\title{
LOW TEMPERATURE ASYMPTOTICS IN SPHERICAL MEAN FIELD SPIN GLASSES
}

\author{
AUKOSH JAGANNATH AND IAN TOBASCO
}

\begin{abstract}
In this paper, we study the low temperature limit of the spherical Crisanti-Sommers variational problem. We identify the $\Gamma$-limit of the Crisanti-Sommers functionals, thereby establishing a rigorous variational problem for the ground state energy of spherical mixed $p$-spin glasses. As an application, we compute moderate deviations of the corresponding minimizers in the low temperature limit. In particular, for a large class of models this yields moderate deviations for the overlap distribution. We then analyze the ground state energy problem. We show that this variational problem is dual to an obstacle-type problem. This duality is at the heart of our analysis. We present the regularity theory of the optimizers of the primal and dual problems. This culminates in a simple method for constructing a finite dimensional space in which these optimizers live for any model. As a consequence of these results, we unify independent predictions of Crisanti-Leuzzi and Auffinger-Ben Arous regarding the 1RSB phase in this limit. We find that the "positive replicon eigenvalue" and "pure-like" conditions are together necessary for optimality, but that neither are themselves sufficient, answering a question of Auffinger and Ben Arous in the negative. We end by proving that these conditions completely characterize the 1RSB phase in $2+p$-spin models.
\end{abstract}

\section{INTRODUCTION}

In this paper, we study the Crisanti-Sommers variational problem which is defined as follows. Let $\xi(t)=\sum_{p \geq 2} \beta_{p}^{2} t^{p}$, which we call the model, and assume that $\xi(1+\epsilon)<\infty$ for some $\epsilon>0$. The Crisanti-Sommers functional is defined for $\mu \in \operatorname{Pr}([0,1])$ by

$$
\mathcal{P}_{\beta, h, \xi}(\mu)=\frac{1}{2}\left(\int_{0}^{1} \beta^{2} \xi^{\prime \prime}(s) \hat{\mu}(s) d s+\int_{0}^{1}\left(\frac{1}{\hat{\mu}(s)}-\frac{1}{1-s}\right) d s+h^{2} \hat{\mu}(0)\right)
$$

where

$$
\hat{\mu}(t)=\int_{t}^{1} \mu[0, s] d s .
$$

Here, $\beta$ is the inverse temperature and $h$ is the external field and they satisfy $\beta>0$ and $h \geq 0$. Note that since $\hat{\mu}(s) \leq 1-s$, the second integral is well-defined. The Crisanti-Sommers variational problem is given by

$$
F(\beta, h, \xi)=\min _{\mu \in \operatorname{Pr}([0,1])} \frac{1}{\beta} \mathcal{P}_{\beta, h, \xi}(\mu) .
$$

For experts: the functional defined above is a lower semi-continuous extension of the functional originally described by Crisanti and Sommers [12]. Its minimization is the same as that of the functional considered in [26]. This is explained in more detail in Section 6.1.

The importance of the Crisanti-Sommers variational problem comes from the study of spherical mixed $p$-spin glasses, which are defined as follows. Let $\Sigma_{N}=S^{N-1}(\sqrt{N})$ and define the Hamiltonian

$$
H_{N}(\sigma)=\beta \sum_{p \geq 2}^{\infty} \frac{\beta_{p}}{N^{\frac{p-1}{2}}} \sum_{i_{1}, \ldots, i_{p}=1}^{N} g_{i_{1} \ldots i_{p}} \sigma_{i_{1}} \cdots \sigma_{i_{p}}+h \sum_{i=1}^{N} \sigma_{i}
$$

Date: September 18, 2018. 
where $g_{i_{1} \ldots i_{p}}$ are i.i.d. $\mathcal{N}(0,1)$ random variables. For the relationship between the study of these problems and the study of the class of smooth, isotropic Gaussian processes on the sphere in high dimension see [1]. Define the partition function and Gibbs measure

$$
Z_{N}=\int_{\Sigma_{N}} e^{H_{N}} d v o l_{N} \quad \text { and } \quad G_{N}(d v o l)=\frac{e^{H_{N}}}{Z_{N}} d v o l_{N}
$$

where $d v^{\circ} l_{N}$ is the normalized volume measure on $\Sigma_{N}$. It was predicted by Crisanti and Sommers [12] and proved by Talagrand [26] and Chen [8] that the thermodynamic limit of the free energy per site is given by the variational formula

$$
\lim \frac{1}{\beta N} \log Z_{N}=\min _{\mu \in \operatorname{Pr}([0,1])} \frac{1}{\beta} \mathcal{P}_{\beta, h, \xi}(\mu) .
$$

The minimizer, $\mu_{\beta, h, \xi}$, is thought of as the order parameter in these systems, and is conjectured to be the limiting law of the overlap, $R_{12}=\left(\sigma^{1}, \sigma^{2}\right) / N$, i.e.,

$$
\mathbb{E}_{N}^{\otimes 2}\left(R_{12} \in A\right) \rightarrow \mu_{\beta, h, \xi}(A) .
$$

This is known, for example, when the collection $\left\{t^{p}: \beta_{p} \neq 0\right\}$ is total in $C([0,1])$ (these are called generic models) 26, 23].

In this paper, we study the zero temperature, i.e., $\beta \rightarrow \infty$, limit of the Crisanti-Sommers variational problem. This limit arises naturally in the method of annealing, an important and nontrivial technique used in the study of random optimization problems [17, 20, 21]. The study of mean field spin glasses is intimately related to the study of random optimization problems in highly disordered energy landscapes. For such problems, it is important to determine the precise asymptotics of the maximum in the limit $N \rightarrow \infty$, i.e.,

$$
\lim _{N \rightarrow \infty} \frac{1}{N} \max _{\sigma \in \Sigma_{N}} H_{N}(\sigma)=G S E .
$$

This quantity is called the ground state energy. As an example, the case where $\xi(t)=t^{2}$ and $h=0$ corresponds to the study of the (renormalized) largest eigenvalue of a GOE random matrix which is well understood. In contrast, the case $\xi(t)=t^{3}$ is much less understood, and corresponds to the study of the maximum of a random trilinear form with Gaussian coefficients. A related and natural question is to study the limiting law of the relative positions of near minimizers in the asympotic that their energies approach the ground state. For a summary of what is known see [1, 25.

The central idea of the method of annealing is that the ground state energy can be computed from the free energy by sending the temperature to zero. In our setting, this means that if $h=\beta h_{0}(\beta)$ and $h_{0} \rightarrow \bar{h}$ as $\beta \rightarrow \infty$, then

$$
G S E=\lim _{\beta \rightarrow \infty} \min _{\mu \in \operatorname{Pr}([0,1])} \frac{1}{\beta} \mathcal{P}_{\beta, h, \xi}(\mu) .
$$

The proof of this result in our setting is standard. (See [1] for a proof in the case that $h_{0}=0$. The case $h_{0} \neq 0$ follows by a straightforward extension of their arguments.) One expects in the annealing limit that

$$
\lim _{\beta \rightarrow \infty} \lim _{N \rightarrow \infty} \mathbb{E} \int f\left(R_{12}\right) d G_{N}^{\otimes 2}\left(R_{12} \in A\right)=f(1) .
$$

As an application of our analysis, we obtain the next order correction to this statement.

Our approach to the zero temperature limit is through $\Gamma$-convergence. This notion was introduced by de Giorgi and is a standard tool in the asymptotic analysis of variational problems. An immediate consequence of the theory that the ground state energy is the minimum value of the $\Gamma$-limit of $\frac{1}{\beta} \mathcal{P}_{\beta, h, \xi}$. A further consequence is that the minimizers at finite $\beta$ converge to the minimizer at $\beta=\infty$, in an appropriate topology. By studying the $\Gamma$-limit as a variational problem unto itself, 
we are able to rule out certain conjectures pertaining to the character of the minimizers at large, but finite, $\beta$.

The zero temperature problem is a strictly convex minimization problem. In principle, one could study its minimizer through its first order optimality conditions. This approach is well-known in the literature surrounding the Parisi variational problem [26, 2, 15]. In this paper, we take an entirely different approach through convex duality. We obtain the convex dual of the zero temperature problem: it is a concave maximization problem of obstacle-type. Obstacle-type problems and their first order optimality conditions, called "variational inequalities", have a long history in the calculus of variations (see e.g. [6, 5, 16]). In these problems, the study of the contact set, the points at which the obstacle and the optimizer are equal, is crucial. In our analysis, we find an interesting connection between the contact set and the choice of model. This connection shares similarities with some results of Cimatti on the shape of a constrained elastic beam [9]. Exploiting this connection, we are able to comment on the phase diagram of the zero temperature problem in full generality.

Determining the full phase diagram of the Crisanti-Sommers variational problem, particularly sharply determining phase boundaries, remains an important and difficult question. The region of $(\beta, h, \xi)$-space in which the minimizer is 1-atomic is known as the RS region, the region in which it is $(k+1)$-atomic is the $k \mathrm{RSB}$ region, and the region in which it is not $k$-atomic for any $k \in \mathbb{N}$ is the FRSB region. A typical question is to find explicit conditions on $(\beta, h, \xi)$ that characterize a $k$ RSB region. In the FRSB region, little is known rigorously about the character of the minimizer. If the minimizer is absolutely continuous on an interval $[a, b]$, then on that interval its density is known (see for example [26, 10]). However, as suggested by the work of [18, 11], one expects that in full generality, the support of the absolutely continuous part may consist of many disjoint intervals. It is interesting to ask if there is a systematic way to reduce the complexity of the space in which the minimizer lives.

These questions have natural analogs when $\beta=\infty$. Though difficult at the level of the primal problem, they are very natural at the level of the dual: they are questions about the topology of the contact set. An isolated atom for the minimizer corresponds to an isolated point in the contact set. An interval in the support of the minimizer corresponds to an interval in the contact set. The question of "how many RSBs" is then "how many connected components does the contact set have". We give a simple method to upper bound the number of connected components. Furthermore, our work gives strong evidence for the predictions of [18, 11]. This scenario runs against the common intuition in the mathematical spin glass community.

The bulk of this paper is regarding these questions. Through our analysis of the $\Gamma$-limit, we describe a general algorithm for producing the minimizer which reduces the problem to a finite dimensional optimization problem. In the case of $2+p$ models, i.e., $\xi(t)=\beta_{2}^{2} t^{2}+\beta_{p}^{2} t^{p}$, we give an exact characterization of the 1RSB region in terms of the coefficients of the model. For general models, these conditions are seen to be necessary. This result rules out the (distinct) characterizations of 1RSB suggested by [10] and [1], and instead proves that, in the case of $2+p$ models, the intersection of these conditions characterizes 1RSB. Our main tool for establishing these results is the new convex duality principle for the limiting functional at $\beta=\infty$.

When a first draft of this paper was complete, we learned of the related work of Chen and Sen 7 . in which the authors also treated the zero temperature limit of the Crisanti-Sommers variational problem. In [7], the authors provided an alternative, but equivalent, variational representation for the ground state energy, obtained the first order optimality conditions and its immediate consequences, then turned to probabilistic questions which, while related, do not overlap with the present work. See Remark 1.5 and the discussion after Theorem 1.10 for more on the relation between these results. 
1.1. Limiting Problem: Gamma convergence results. Our first result establishes a variational representation for the ground state energy. We begin by introducing the topological space

$$
\mathcal{A}=\left\{\nu \in \mathscr{M}([0,1]): \nu=m(t) d t+c \delta_{1}, m(t) \geq 0 \text { is non-decreasing and cadlag }\right\}
$$

equipped with the relative topology induced by the weak-* topology on $\mathscr{M}([0,1])$, the space of finite measures on $[0,1]$, i.e., the topology of weak convergence of measures. In the subsequent, $m(t)$ will always refer to the unique representative of the density of $\nu$ that satisfies the above conditions and is left-continuous at 1.

On the space $\mathcal{A}$, we define the subsets

$$
X_{\beta}=\{\nu \in \mathcal{A}: d \nu=\beta \mu[0, t] d t, \quad \mu \in \operatorname{Pr}[0,1]\},
$$

and we lift the functional $\frac{2}{\beta} \mathcal{P}_{\beta, h, \xi}$ to $\mathcal{A}$ as $F_{\beta, h, \xi}: \mathcal{A} \rightarrow[0, \infty]$,

$$
F_{\beta, h, \xi}(\nu)=\left\{\begin{array}{ll}
\frac{2}{\beta} \mathcal{P}_{\beta, h, \xi}(\mu) & \nu \in X_{\beta} \\
\infty & \nu \notin X_{\beta}
\end{array} .\right.
$$

Finally, we define the functional $G S_{h, \xi}: \mathcal{A} \rightarrow[0, \infty]$ as

$$
G S_{h, \xi}(\nu)=\left\{\begin{array}{ll}
\int_{0}^{1} \xi^{\prime \prime}(s) \nu[s, 1]+\frac{1}{\nu[s, 1]} d s+h^{2} \nu[0,1] & \nu \neq 0 \\
\infty & \nu=0
\end{array} .\right.
$$

Observe that $G S$ has a unique minimizer. Indeed, it is strictly convex by the strict convexity of $x \rightarrow \frac{1}{x}$ and sequentially lower semi-continuous by Fatou's lemma. Furthermore, the sets $\{G S(\nu) \leq C\}$ for $C<\infty$ are sequentially compact in $\mathcal{A}$ by Lemma 6.3 applied with $f=\xi^{\prime \prime}$.

Before we state our first result, we remind the reader of the notion of sequential $\Gamma$-convergence [3].

Definition 1.1. Let $X$ be topological space. We say that a sequence of functionals $F_{n}: X \rightarrow$ $[-\infty, \infty]$ sequentially $\Gamma$-converges to $F: X \rightarrow[-\infty, \infty]$ if

(1) The $\Gamma-\lim$ inf inequality holds: for every $x$ and every sequence $\lim _{n \rightarrow \infty} x_{n}=x$

$$
\liminf _{n} F_{n}\left(x_{n}\right) \geq F(x)
$$

(2) The $\Gamma-\lim$ sup inequality holds: for every $x$ there is a sequence $\lim _{n \rightarrow \infty} x_{n}=x$ such that

$$
\limsup _{n \rightarrow \infty} F_{n}\left(x_{n}\right) \leq F(x)
$$

We denote this by $F_{n} \stackrel{\Gamma}{\rightarrow} F$. For a sequence of functionals indexed by a real parameter $\beta$ we say that $F_{\beta} \stackrel{\Gamma}{\rightarrow} F$ if for every subsequence $\beta_{n} \rightarrow \infty, F_{\beta_{n}} \stackrel{\Gamma}{\rightarrow} F$.

Remark 1.2. The following remark will only be of interest to experts in the field of $\Gamma$-convergence. We observe here that the notion of sequential $\Gamma$-convergence is distinct from the notion of $\Gamma$ convergence in our setting as we are not working in a metrizable space. For a brief discussion of this see [3, 13. Nevertheless, the usual consequences of $\Gamma$-convergence carry through to our setting in the sequential case. We place the proof of those results that we use in the appendix.

Theorem 1.3. Suppose that $\left(\beta, h, \xi_{\beta}\right)$ are such that $\frac{h}{\beta} \rightarrow \bar{h}$ and $\xi_{\beta}^{\prime \prime} \rightarrow \xi^{\prime \prime}$ uniformly as $\beta \rightarrow \infty$. Then,

$$
F_{\beta, h, \xi_{\beta}} \stackrel{\Gamma}{\rightarrow} G S_{\bar{h}, \xi}
$$


In particular,

$$
G S E=\min _{\nu \in \mathcal{A}} \frac{1}{2} G S_{h, \xi}(\nu)
$$

Furthermore, we have that if $\nu_{\beta}$ are the (unique) minimizers of $F_{\beta, h, \xi_{\beta}}$ then

$$
\nu_{\beta} \stackrel{w-*}{\longrightarrow} \nu
$$

where $\nu$ is the unique minimizer of $G S_{h, \xi}$.

As explained above, an an immediate corollary of the $\Gamma$-convergence is a moderate deviation principle in the limit $\beta \rightarrow \infty$ for the minimizers.

Corollary 1.4. Let $\nu=m(t) d t+c \delta_{1} \in \mathcal{A}$ be the unique minimizer of $G S_{h, \xi}$, and $\mu_{\beta}$ be the (unique) minimizers of $\mathcal{P}_{\beta, h, \xi_{\beta}}$ where $\left(\beta, h, \xi_{\beta}\right)$ satisfy the conditions of Theorem 1.3. Then,

(1) For any $f \in C^{1}$,

$$
\lim \beta\left[f(1)-\int f d \mu_{\beta}\right]=\int f^{\prime} d \nu
$$

(2) For every $t<1$ that is a continuity point of $m(t)$,

$$
\beta \mu_{\beta}[0, t] \rightarrow m(t)
$$

(3) Let $q_{\beta} \rightarrow 1$ be such that $\beta \mu_{\beta}\left[0, q_{\beta}\right) \rightarrow m\left(1^{-}\right)$, and suppose that $m\left(1^{-}\right)<\infty$. Then if $Y_{\beta}$ have law $\mu_{\beta}$, it follows that

$$
\nu(\{1\})=\lim \mathbb{E}_{\mu_{\beta}}\left(\beta\left(1-Y_{\beta}\right) \mid Y_{\beta} \in\left[q_{\beta}, 1\right]\right) .
$$

Remark 1.5. In [7], Chen and Sen also obtained (1.1.3). In their notation $\nu([0,1])=L$ and $m(t)=\alpha_{0}(t)$. The convergence results stated there are equivalent to Corollary $1.4(2)$, combined with the convergence of $\nu_{\beta}([0,1])$ to $\nu([0,1])$. The strict inequality between $L$ and $\int \alpha_{0}$ in [7] will follow from Theorem 1.11 .

Remark 1.6. An immediate consequence of this result is a moderate deviation principle for the overlap distribution, $R_{12}$, for models for which $\mu_{\beta}$ is known to be its limiting law (e.g. generic models). By a standard differentiation and convexity argument (see, e.g., [23, Theorems 3.7, 3.8]), the Gibbs measure concentrates on the set $\left\{\left|H_{N} / N-\mathbb{E}\left\langle H_{N}\right\rangle / N\right|<\epsilon\right\}$ in the thermodynamic limit for all $\epsilon>0$. By a standard integration by parts argument (see also [26, Theorem 1.2]), $\lim _{N} \mathbb{E}\left\langle H_{N}\right\rangle / N=\int(\xi(1)-\xi(t)) \beta d \mu_{\beta}$. These results, along with those in Corollary 1.4, yield asymptotic information about the law of the relative positions of near maximizers of $\overline{H_{N}}$ in the large $N$, large $\beta$ limit.

Remark 1.7. As we shall soon see, $m\left(1^{-}\right)<\infty$ in our setting (see Theorem 1.11). We note, however, that it is not true that $\beta \mu_{\beta} \rightarrow d m$ weakly as measures, since $\beta \mu_{\beta}([0,1])=\beta \rightarrow \infty$.

Remark 1.8. One has to be careful interpreting (3) for the following reason: it may be that $\beta\left(1-q_{\beta}\right)$ explodes. This is neither prevented by the convergence on $\mathcal{A}$ nor by finite energy considerations. The following is an interesting example to keep in mind. Let $\beta \mu_{\beta}=m \delta_{q_{1}}+(1-m) \delta_{q_{2}}$ where $q_{1}=1-\frac{1}{\sqrt{\beta}}, q_{2}=1-\frac{1}{\beta}$, and $m=\sqrt{\beta}$. Then $\int_{s}^{1} \beta \mu_{\beta} d t \sim 2+O\left(\frac{1}{\sqrt{\beta}}\right)$ and the corresponding energy scales like $\frac{1}{2}+\frac{1}{\sqrt{\beta}} \log (2)+O\left(\frac{1}{\beta}\right)$ which is finite. A similar example can be constructed to show that a quantification of the rate at which $q_{*}(\beta)=\sup \operatorname{supp} \mu_{\beta} \rightarrow 1$ is out of the reach of these methods as the topology of these results are too weak (though clearly $\lim \sup \beta\left(1-q_{*}(\beta)\right) \leq$ $\left.\limsup \int_{0}^{1} \beta \mu_{\beta}([0, t]) d t \leq C\right)$. We believe that one would require sharp estimates on $q_{*}$, such as might come from the first order optimality conditions, in order to obtain such a result. 
Example 1.9. Let $h=0$. Suppose that the minimizers $\mu_{\beta}$ are $1 R S B$. Then we have that $\beta\left(1-q_{*}\right) \rightarrow \nu\{1\}$ and $\beta \mu_{\beta}(\{0\}) \rightarrow \nu([0,1))$.

The proofs of these results are in Section 2,

1.2. Convex Duality Results. We turn now to the analysis of the limiting variational problem. First, we find it convenient to make the following change of variables. Define

$$
\mathcal{C}=\{\phi \in C([0,1]): \phi \geq 0, \phi \text { is non-increasing, } \phi \text { is concave }\}
$$

and define the functional $P_{h, \xi}: \mathcal{C} \rightarrow[0, \infty]$ by

$$
P_{h, \xi}(\phi)=\int \xi^{\prime \prime} \phi+\frac{1}{\phi} d x+h^{2} \phi(0) .
$$

Observe that the space $\mathcal{A}$ and the space $\mathcal{C}$ are in one-to-one correspondence. In particular, every $\phi \in \mathcal{C}$ is of the form $\nu[s, 1]$ for some $\nu \in \mathcal{A}$ and similarly every $\nu \in \mathcal{A}$ is of the form $\phi^{\prime}(t) d t+\phi(1) \delta_{1}$ for some $\phi \in \mathcal{C}$. Here, the derivative $\phi^{\prime}$ is understood distributionally and is an element of $L^{1}$. This correspondence and other important results about these spaces are summarized in Section 6.2. In particular, observe that $G S(\nu)=P(\phi)$ whenever $\nu$ and $\phi$ are in correspondence, so that

$$
G S E=\min _{\phi \in \mathcal{C}} \frac{1}{2} P_{h, \xi}(\phi) .
$$

Our main tool in the analysis of the limiting variational problem is an important duality principle, which relates this problem to a one-dimensional variational problem of obstacle-type. To define it, let

$$
\mathcal{K}_{h, \xi}=\left\{\eta \in C([0,1]): \eta \geq \xi, \eta(1)=\xi(1), \eta^{\prime}(0)=\xi^{\prime}(0)-h^{2}, \eta \text { is convex }\right\},
$$

equipped with the norm topology. Basic properties of this space are summarized in Section 6.2. In particular, $\eta^{\prime} \in B V((0,1))$ so that $\eta^{\prime}$ has well-defined trace at 0 . Furthermore, $\eta^{\prime \prime}$ can be uniquely represented by $\mu \in \mathscr{M}([0,1])$ with $\mu(\{0,1\})=0$. In the following, $\eta^{\prime \prime}$ will always refer to this representative.

Now define the functional $D: \mathcal{K}_{h, \xi} \rightarrow[0, \infty)$,

$$
D(\eta)=2 \int \sqrt{\eta_{a c}^{\prime \prime}(x)} d x
$$

Here for a measure $\nu$, we let $\nu_{a c}(x)=\frac{\partial \nu}{\partial \mathcal{L}}$ be its density with respect to $\mathcal{L}$, the Lebesgue measure. Basic properties of $D$ are proved in Section 6.3. In particular, by Corollary 6.15, it is upper semi-continuous.

We then have the following duality principle:

Theorem 1.10. We have that

$$
\min _{\phi \in \mathcal{C}} P_{h, \xi}(\phi)=\max _{\eta \in \mathcal{K}_{h, \xi}} D(\eta) .
$$

Furthermore, the optimizers satisfy

$$
\phi^{2} \eta^{\prime \prime}(d x)=d x
$$

These results are proved in Section 3. Problems of the type

$$
G S E=\max _{\eta \in \mathcal{K}_{h, \xi}} D(\eta)
$$

are called obstacle problems and have a rich literature. The obstacle problem approach to studying variational problems on the space of measures has become an important tool, see for example [24]. 
Before turning to the analysis of the primal-dual pair $(P, D)$, we wish to comment briefly on the relationship between our approach to optimality and that which concerns the primal problem alone. Consider the first order optimality conditions for the primal problem at $h=0: \phi \in \mathcal{C}$ is optimal if and only if

$$
\left(\xi^{\prime \prime}-\frac{1}{\phi^{2}}, \psi-\phi\right) \geq 0 \quad \forall \psi \in \mathcal{C} .
$$

This variational inequality and others like it play an essential role in the analysis of Parisi measures (see e.g. [26, 2, 15, 7]). In this approach, the difficulty is to prove that a certain function on $[0,1]$ depending on the choice of measure is minimized on the support of said measure. (In our work, $d m=-\phi^{\prime \prime}$ plays the role of this measure.) The duality between $P$ and $D$, however, suggests an entirely different approach, namely the simultaneous analysis of the variables $\phi$ and $\eta$. The optimal pair, $(\phi, \eta)$, not only achieves the equality $P(\phi)=D(\eta)$, it is characterized by it. Furthermore, the variational inequality 1.2 .7 is implied by this observation. This simultaneous analysis is the crux of our approach.

We now present an analysis of the optimizers. We begin by discussing their regularity.

Theorem 1.11. Let $\phi \in \mathcal{C}$ and $\eta \in \mathcal{K}_{h, \xi}$ be optimal for $P_{\xi}$ and $D$ respectively. Then,

(1) For $\phi$ :

(a) There is a $c>0$ such that $0<c \leq \phi$.

(b) $\phi^{\prime} \in L^{\infty}$.

(2) For $\eta$ :

(a) $\eta \in C^{2}([0,1])$

(b) $\frac{1}{\sqrt{\eta^{\prime \prime}}} \in C([0,1])$, has distributional derivative $\left(\frac{1}{\sqrt{\eta^{\prime \prime}}}\right)^{\prime} \in L^{1}$ which is monotone decreasing, and has second distributional derivative

$$
\left(\frac{1}{\sqrt{\eta^{\prime \prime}}}\right)^{\prime \prime}=-\mu
$$

for some non-negative Radon measure on $(0,1)$. Furthermore supp $\mu \subset\{\xi=\eta\}$.

(c) On the set $\{\xi=\eta\}$,

(i) $\eta^{\prime}(t)=\xi^{\prime}(t)$

(ii) $\eta^{\prime \prime}(t) \geq \xi^{\prime \prime}(t)$

(d) (Natural Boundary Conditions) We have that

(i) $\eta^{\prime}(1)=\xi^{\prime}(1)$

(ii) $\eta(0)=\xi(0)$ or $\phi^{\prime}(0)=0$

(3) For $\mu$ : we have that

$$
\phi^{\prime \prime}=\left(\frac{1}{\sqrt{\eta^{\prime \prime}}}\right)^{\prime \prime}=-\mu
$$

as elements of $\mathcal{D}^{\prime}$. Moreover, $\mu$ is a finite measure.

Since the dual problem is an obstacle-type problem, the following definition is natural.

Definition 1.12. A point $t \in[0,1]$ such that $\eta(t)=\xi(t)$ is called a contact point. The set of contact points is called the contact set.

We then have the following regularity result concerning the contact set of this obstacle problem.

Theorem 1.13. Let

$$
\mathfrak{d}(t)=\left(\frac{1}{\sqrt{\xi^{\prime \prime}}}\right)^{\prime \prime}(t)
$$

Then we have the following two cases: 


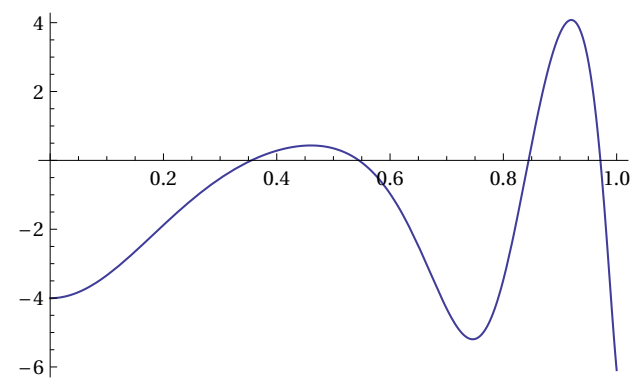

Figure 1. This plot shows the function $\mathfrak{d}$ from Theorem 1.13 for the model $\xi(t)=$ $\frac{300}{601} t^{2}+\frac{200}{601} t^{4}+\frac{100}{601} t^{15}+\frac{1}{601} t^{60}$. The intervals on which $\mathfrak{d}$ is negative are compatible with FRSB. The intervals on which $\mathfrak{d}$ is positive permit at most two atoms each. As shown in Example 1.15, this can be used to reduce the primal problem to a certain finite dimensional optimization problem, that depends on the particular arrangement of these intervals.

(1) If $\mathfrak{d}(t)>0$ on $(a, b)$, then there are at most two contact points in $[a, b]$.

(2) If $\mathfrak{d}(t) \leq 0$ on $[a, b]$, then if there are two contact points $t_{1}, t_{2} \in[a, b]$, then $\left[t_{1}, t_{2}\right] \subset\{\eta=\xi\}$.

Remark 1.14. The finite temperature analogue of part 1 of this result can be seen in [10] as observed in [26].

This result provides us with a systematic dimension reduction which reduces the analysis of 1.2.3 to a finite dimensional optimization problem. Rather than describing this at the level of generalities, we prefer to illustrate these ideas through three examples. In each, we think of building up the ansatz on connected components of the sets

$$
N=\{t \in[0,1]: \mathfrak{d} \leq 0\} \quad \text { and } \quad P=\{t \in[0,1]: \mathfrak{d} \geq 0\} .
$$

For simplicity, we work with $h=0$.

Example 1.15. Let $\xi(t)=\frac{300}{601} t^{2}+\frac{200}{601} t^{4}+\frac{100}{601} t^{15}+\frac{1}{601} t^{60}$. The corresponding $\mathfrak{d}$ is depicted in Figure 1 on page 8. Evidently, $\mathfrak{d}(t)=0$ has exactly four solutions in $[0,1]$, which we label as $0<r_{1}<r_{2}<r_{3}<r_{4}<1$. We call $r_{0}=0$ and $r_{5}=1$. Now $N=\left[r_{0}, r_{1}\right] \cup\left[r_{2}, r_{3}\right] \cup\left[r_{4}, r_{5}\right]$ and $P=\left[r_{1}, r_{2}\right] \cup\left[r_{3}, r_{4}\right]$. By Theorem 1.13 , each component of $N$ can intersect the contact set in at most one closed interval (possibly degenerate). Each component of $P$ can intersect the contact set in at most two points. This motivates the following construction. Let $q_{i, j} \in\left[r_{i-1}, r_{i}\right]$ and $m_{i, j} \in[0, \infty)$ for $i=1, \ldots, 4, j=1,2$. Assume that $q_{i, 1} \leq q_{i, 2}$ for all $i$. Then, by the results above, the optimal $\phi$ must be of the form $\phi=\int_{t}^{1} \mu[0, s] d s+c$ where

$$
\mu=\sum_{i} m_{i, 1} \delta_{q_{i, 1}}+m_{i, 2} \delta_{q_{i, 2}}-\sum_{i: I_{i} \subset N} \mathfrak{d} \cdot \mathbb{1}_{\left[q_{i, 1}, q_{i, 2}\right]} d x
$$

and $c \in(0, \infty)$. In fact, $q_{1,1}=0$ by the same argument as in the proof of Lemma 1.20 .

The next examples are comparatively straightforward. The reader will observe that the key simplification comes from the fact that either $N=\emptyset$ or $P=\emptyset$.

Example 1.16. Let $\xi(t)=\sinh (t)$. Then $\mathfrak{d}(t)=8(5+\cosh 2 t)(\sinh t)^{-5 / 2}$ and $P=[0,1]$. Hence, by Theorem 1.13 , there can be at most two contact points in $[0,1]$. We already know that 1 is a contact point, and by the same argument as in the proof of Lemma 1.20 we can show that 0 must be a contact point as well. Therefore the optimal $\phi$ must be of the form $\phi=m(1-t)+c$ where $m, c>0$. (Since $\xi \neq \xi_{R S}$ we know that $m \neq 0$ by Lemma 1.19.) 
Example 1.17. Let $\xi(t)=\frac{14}{15} t^{2}+\frac{1}{15} t^{4}$. Then $\mathfrak{d}(t)=\frac{3 \sqrt{15}}{2}\left(6 t^{2}-7\right)\left(3 t^{2}+7\right)^{-5 / 2}$ and $N=[0,1]$. Hence, by Theorem 1.13 , if there are two contact points $a, b \in[0,1]$ then $[a, b] \subset\{\eta=\xi\}$. Now 1 is a contact point, and by the same argument as in the proof of Lemma 1.20 we can show that 0 must be a contact point as well. Therefore the optimal $\eta$ must be $\eta=\xi$.

The proofs of these results are given in Section 3 and Section 4 .

1.3. Application to the Analysis of Phase Transitions. The notions of RS, RSB, kRSB, and FRSB have natural extensions to $\beta=\infty$. In this section, we define these extensions and apply the methods of the previous section to study the 1RSB class in detail. In the case of $2+p$ models, we characterize 1RSB exactly. For simplicity, we will assume that $h=0$ throughout the remainder of the introduction. We use the shorthand $P_{\xi}=P_{0, \xi}$ and $\mathcal{K}_{\xi}=\mathcal{K}_{0, \xi}$.

1.3.1. Definition of $k R S B$. The following is an elementary consequence of the $\Gamma$-convergence.

Lemma 1.18. Let $\left(\beta, h, \xi_{\beta}\right)$ satisfy the assumptions of Theorem 1.3, and assume that $\frac{h}{\beta}=o_{1}(\beta)$. Suppose that there are $k \in \mathbb{N}, \beta_{c} \in \mathbb{R}_{+}$such that for all $\beta \geq \beta_{c}$, the minimizer $\mu_{\beta}$ of $\mathcal{P}_{\beta, h, \xi_{\beta}}$ is $k$ atomic. Then the minimizer $\nu=m(t) d t+c \delta_{1}$ of $G S_{0, \xi}$ is such that $d m$ is at most $k-1$ atomic on $[0,1)$.

With this and the correspondence $\mathcal{A} \leftrightarrow \mathcal{C}$ in mind, we define for each $k \in \mathbb{N}$ the set

$$
R S B_{k}=\{\phi \in \mathcal{C}: d m \text { is } k-\text { atomic on }[0,1)\}
$$

and, with slight abuse of notation, we call $k R S B$ the set of models such that the optimal $\phi$ for $P_{\xi}$ is in $R S B_{k}$. Similarly, we call $R S$ the set of models such that the optimal $\phi$ is constant. We call $R S B$ the complement of this, and we call $F R S B$ the region where the optimal $d m$ is neither zero nor $k$-atomic for any $k \in \mathbb{N}$.

In the ground state problem at zero external field, RS is particularly simple.

Lemma 1.19. $R S=\left\{\xi_{S K}\right\}$.

Proof. Applying the natural boundary conditions from Theorem 1.11 to $\eta$, we see that $\phi=c$ yields $c^{2}=\frac{1}{\xi^{\prime}(1)}$, so that $\eta$ must be of the form

$$
\eta(t)=\xi(1)+\xi^{\prime}(1) \frac{t^{2}-1}{2} .
$$

By the same regularity theorem, $\eta^{\prime \prime}(1) \geq \xi^{\prime \prime}(1)$ so that $\xi^{\prime}(1) \geq \xi^{\prime \prime}(1)$. This implies that $\xi=\xi_{S K}$. On the other hand, if $\xi=\xi_{S K}$, then $\eta$ given by 1.3.1) is in $\mathcal{K}_{h, \xi}$ and maximizes $D$ so that the optimal $\phi$ is constant.

The next result establishes the existence of an "atom at zero" at $\beta=\infty$.

Lemma 1.20. If $\xi \in k R S B$, then the optimal $\phi$ for $P_{\xi}$ satisfies $\phi^{\prime}(0)>0$. Equivalently, dm has an atom at zero.

Proof. Suppose that $q \in(0,1)$ is an atom of $d m, \phi^{\prime \prime}=0$ in $(0, q)$, and $\phi^{\prime}(0)=0$. Then $q$ is a contact point of $\eta$, and $\phi$ is constant on $(0, q)$. Hence, by Theorem 1.11, the optimal $\eta$ is of the form $\eta(t)=a+b t+c t^{2}$ for $t \in(0, q)$. By that same theorem, $\eta^{\prime}(0)=\xi^{\prime}(0), \eta(q)=\xi(q)$, and $\eta^{\prime}(q)=\xi^{\prime}(q)$. Thus,

$$
\eta(t)=\xi(q)-\frac{\xi^{\prime}(q) q}{2}+\frac{\xi^{\prime}(q)}{2 q} t^{2} \quad t \in(0, q) .
$$

However, since $q$ is a contact point, $\xi^{\prime \prime}(q) \leq \eta^{\prime \prime}(q)$ and hence $\xi^{\prime \prime}(q) \leq \frac{\xi^{\prime}(q)}{q}$. This can only happen if $\xi=\xi_{S K}$. 
1.3.2. Characterization of $1 R S B$. In this section, we will study the special case that the minimizer is $1 \mathrm{RSB}$. We re-define $R S B_{1}$ to be

$$
R S B_{1}=\{\phi: \phi(t)=c+m(1-t), m, c \in(0, \infty)\} .
$$

This slight abuse of notation is justified by Lemma 1.20 since we are at zero external field. We will refer only to this definition for this section.

The 1RSB region has been studied in the physics and mathematics literature through different techniques. This lead to different proposed characterizations of the 1RSB region. Auffinger and Ben Arous conjectured a criterion for when a model should be $1 R S B$, which they call pure-like [1]. Let $\nu=\xi(1)=1, \nu^{\prime}=\xi^{\prime}(1)$ and $\nu^{\prime \prime}=\xi^{\prime \prime}(1)$, and define

$$
A B A(\xi)=A B A\left(\nu^{\prime}, \nu^{\prime \prime}\right)=\log \left(\frac{\nu^{\prime \prime}}{\nu^{\prime}}\right)-\left(\frac{\left(\nu^{\prime \prime}-\nu^{\prime}\right)\left(\nu^{\prime \prime}-\nu^{\prime}+\nu^{\prime 2}\right)}{\nu^{\prime \prime} \nu^{\prime 2}}\right) .
$$

Definition 1.21. A model $\xi$ is called pure-like if $A B A>0$, critical if $A B A=0$, and full-like if $A B A<0$.

Separately, Crisanti and Leuzzi [10] predicted that the model is 1RSB provided the 1RSB Replicon Eigenvalue is positive. To define this, we introduce for $\phi \in \mathcal{C}$ the formal conjugate

$$
\eta(t)=\xi(1)-R(t)
$$

where

$$
R(t)=\int_{t}^{1} \int_{0}^{s} \frac{1}{\phi^{2}(\tau)} d \tau d s .
$$

(In the case that $h \neq 0$, this formula would have an extra term.)

Definition 1.22. Let $\xi \neq \xi_{S K}$ and let $m, c>0$ solve (5.1.1). Let $\eta$ be the formal conjugate to $\phi=m(1-t)+c$. The quantity $\eta^{\prime \prime}(0)-\xi^{\prime \prime}(0)$ is called the 1RSB Replicon eigenvalue or simply the replicon eigenvalue.

A consequence of Theorem 1.11 is that in order for a model to be 1RSB, it must be both pure-like and have positive replicon eigenvalue.

Corollary 1.23. Suppose that the optimal $\phi \in R S B_{1}$. Then necessarily,

(1) $m, c$ solve (5.1.1);

(2) The model is pure-like or critical: $\eta^{\prime \prime}(1) \geq \xi^{\prime \prime}(1)$;

(3) The replicon eigenvalue is non-negative: $\eta^{\prime \prime}(0) \geq \xi^{\prime \prime}(0)$.

Remark 1.24. Let $\left(\beta, h, \xi_{\beta}\right)$ satisfy the assumptions of Theorem 1.3, and assume that $\frac{h}{\beta}=o_{1}(\beta)$. Then as a consequence of this result and the convergence of minimizers from the $\Gamma$-convergence, we see that both conditions (2) and (3) must be met for $\left(\beta, h, \xi_{\beta}\right)$ to be 1RSB for large $\beta$.

That being said, we find that in full generality, neither of these conditions are themselves sufficient for optimality. For example, models of the form $\xi(t)=\mu t^{2}+(1-\mu) t^{4}$ are pure-like provided $\mu<\mu_{c} \approx$.786444. However, for the choice $\mu=.7$, the formal conjugate $\eta$ to $\phi=m(1-t)+c$, where $m, c$ solve (5.1.1), satisfies $\eta(t)-\xi(t)<0$ for $t<.4$. Hence, this model is not 1RSB. Similarly, a model satisfying $\xi^{\prime \prime}(0)=0$ always has non-negative replicon eigenvalue. Therefore it suffices to find a model with no $p=2$ term which is full-like. As described in [1, Fig. 2], there exists $\mu \in(0,1)$ such that $\xi(t)=\mu t^{4}+(1-\mu) t^{30}$ is full-like.

The analysis of $1 \mathrm{RSB}$ in the specific case of $2+p$ models is of particular interest to the spin glass community. These are models of the form $\xi(t)=\mu t^{2}+(1-\mu) t^{p}, \mu \in[0,1]$. In this setting, we resolve the $1 \mathrm{RSB}$ conjectures. 
Theorem 1.25. Let $\xi$ be $a 2+p$ model other than $\xi_{S K}$. Then $\xi \in 1 R S B_{\infty}$ if and only if both the replicon eigenvalue is non-negative and the model is pure-like or critical.

Remark 1.26. It is interesting to note that the essential difficulties in proving a result of this type, namely proving the obstacle condition, bears a striking resemblance to testing the validity of the second moment method approach of Subag [25].

We close this introduction by noting the following curiosity: one may be tempted to conjecture that the result of Theorem 1.25 holds for general models. Such a result would have to rest crucially on the assumption that the power series $\xi$ has non-negative coefficients. To see this, let $\xi$ be a 1RSB model and let $\phi$ be optimal for $P$. Of course, $\phi \in R S B_{1}$, and its formal conjugate $\eta$ is optimal for $D$. Note that none of the arguments leading up to (5.1.1) require that $\xi$ is a power series with positive coefficients. Furthermore, only $\xi(1)$ and $\xi^{\prime}(1)$ are required to determine $m_{*}, c_{*}$ by (5.1.1). Thus, if we change $\xi$ by adding a bump function which is supported away from 0 and $1, \phi$ must still be the $R S B_{1}$ ansatz as above. Evidently, we can arrange for the altered $\xi$ to not satisfy the obstacle condition $\eta \geq \xi$. Thus, the positivity of the coefficients in the power series of $\xi$ is crucial to the validity of theorems of the form Theorem 1.25 .

The results from this section are proved in Section 5 .

1.4. Acknowledgments. We would like to thank our advisors G. Ben Arous and R.V. Kohn for their support. A.J. would like to thank E. Subag and O. Zeitouni for helpful discussions regarding the presentation of this work. We would like to thank the New York University GRI Institute in Paris for its hospitality during the initial phase of this project. A.J. would like to thank the Department of Mathematics at Northwestern University for its hospitality during the preparation of this paper. This research was conducted while A.J. was supported by a National Science Foundation Graduate Research Fellowship DGE-0813964; and National Science Foundation grants DMS-1209165 and OISE-0730136, and while I.T. was supported by a National Science Foundation Graduate Research Fellowship DGE-0813964; and National Science Foundation grants OISE-0967140 and DMS-1311833.

1.5. Notation and Spaces. The notation $f_{I} f$ denotes the average of $f$ over $I . \partial_{+} f$ and $\partial_{-} f$ are respectively the right and left derivative. $C_{l o c}^{k}((0,1))$ is the space of functions that are $C^{k}$ on every compact subset of $(0,1)$ and $W^{k, p}((0,1))$ is the Sobolev space of functions on $(0,1)$ that are $k$ times weakly differentiable with derivatives lying in $L^{p}$. The space $\mathcal{D}=C_{c}^{\infty}((0,1))$ is the usual space of test functions and $\mathcal{D}^{\prime}$ denotes the space of distributions. $B V=B V((0,1))$ is the space of bounded variation functions, i.e., those $f \in L^{1}$ with first distributional derivative $f^{\prime}$ given by finite signed measures on $(0,1) . \mathscr{M}=\mathscr{M}([0,1])$ is the space of finite signed measures on $[0,1] . \mathscr{M}_{+} \subset \mathscr{M}$ are those measures that are non-negative.

\section{Gamma convergence Results}

We begin this section by proving Theorem 1.3 . We then turn to proving Corollary 1.4 .

2.1. The functional convergence. In the following two lemmas we fix $\xi_{\beta}=\xi$ and $h=0$ and let $F_{\beta}=F_{\beta, 0, \xi}$.

We begin with the proof of the $\Gamma$-liminf inequality.

Lemma 2.1. ( $\Gamma$-liminf.) If $\left(\nu_{\beta}\right) \in \mathcal{A}$ converges $\nu_{\beta} \rightarrow \nu$ weakly, then

$$
\liminf F_{\beta}\left(\nu_{\beta}\right) \geq G S(\nu) \text {. }
$$

Proof. Without loss of generality, assume that $\left\{\nu_{\beta}\right\}$ satisfies $F_{\beta}\left(\nu_{\beta}\right) \leq C$. This implies that $\nu_{\beta} \in$ $X_{\beta}$, so that $d \nu_{\beta}=\beta \mu_{\beta}[0, t] d t$ for some $\mu_{\beta} \in \operatorname{Pr}([0,1])$, and 


$$
\liminf F_{\beta}\left(\nu_{\beta}\right)=\liminf \int_{0}^{1} \xi^{\prime \prime}(s) \beta \hat{\mu}_{\beta}(s) d s+\int_{0}^{1} \frac{1}{\beta}\left(\frac{1}{\hat{\mu}(s)}-\frac{1}{1-s}\right) d s=\liminf I_{\beta}+I I_{\beta} .
$$

By properties of weak convergence, $\lim \nu_{\beta}[s, 1]=\nu[s, 1], \mathcal{L}$ - a.e., from which it follows that the first term converges,

$$
\lim I_{\beta}=\int_{0}^{1} \xi^{\prime \prime}(s) \nu[s, 1] d s
$$

by the bounded convergence theorem. Now consider the second term. By Fatou's lemma,

$$
\liminf I I_{\beta} \geq \int_{0}^{1} \liminf \frac{1}{\nu_{\beta}[s, 1]}\left(1-\frac{1}{\beta} \frac{\nu_{\beta}[s, 1]}{1-s}\right) d s=\int_{0}^{1} \frac{1}{\nu[s, 1]} d s
$$

Therefore,

$$
\liminf F_{\beta}\left(\nu_{\beta}\right) \geq G S(\nu)
$$

as required.

Lemma 2.2. (T-limsup.) For every $\nu \in \mathcal{A}$, there exists a sequence $\left\{\nu_{\beta}\right\} \subset \mathcal{A}$ such that $\nu_{\beta} \rightarrow \nu$ weakly and

$$
\limsup F_{\beta}\left(\nu_{\beta}\right) \leq G S(\nu) .
$$

Proof. Write $d \nu=m(t) d t+c \delta_{1}$ and define

$$
c_{\beta}= \begin{cases}c & c>0 \\ \frac{1}{\beta} & c=0\end{cases}
$$

Claim 2.3. For $\beta>\nu[0,1]+1$, there exists a $q_{\beta} \in(0,1)$ with the following properties

(1) $\int_{q_{\beta}}^{1} m(t) d t+c_{\beta}=\beta\left(1-q_{\beta}\right)$.

(2) $q_{\beta} \rightarrow 1$

(3) $\frac{m\left(q_{\beta}\right)}{\beta} \leq 1$

Proof. To see (1), let $f(t)=\int_{t}^{1} m(t) d t+c_{\beta}$ and $g(t)=\beta(1-t)$. Then

$$
g(0)=\beta>\nu[0,1]+1>\int_{0}^{1} m(t) d t+c_{\beta}=f(0)
$$

and

$$
0=g(1)<f(1)=c_{\beta} .
$$

The result then follows by the intermediate value theorem. Choose any such $q_{\beta}$.

To see (2), observe that

$$
0 \leq \beta\left(1-q_{\beta}\right) \leq \int_{0}^{1} m(t) d t+c_{\beta} \leq C(\nu)
$$

To see (3), observe that by (1),

$$
f_{q_{\beta}}^{1} \frac{m(t)}{\beta} d t \leq 1 .
$$

Since $m$ is non-decreasing, (3) follows. 
Let $\beta$ and $q_{\beta}$ be as in the above claim and let

$$
\mu_{\beta}[0, t]= \begin{cases}\frac{m(t)}{\beta} & t<q_{\beta} \\ 1 & t \geq q_{\beta}\end{cases}
$$

Note that this defines a probability measure so that $\mu_{\beta} \in \operatorname{Pr}[0,1]$. This gives us $d \nu_{\beta}=\beta \mu_{\beta}[0, t] d t \in$ $X_{\beta}$

First observe that $\nu_{\beta} \rightarrow \nu$. To see this, observe that if $\phi \in C([0,1])$,

$$
\begin{aligned}
\int \phi d \nu_{\beta} & =\int_{0}^{q_{\beta}} \phi m d t+\int_{q_{\beta}}^{1} \beta \phi(t) d t \\
& =\int_{0}^{q_{\beta}} \phi m d t+\left(\int_{q_{\beta}}^{1} m d t+c_{\beta}\right) f_{q_{\beta}}^{1} \phi(t) \rightarrow \int \phi m d t+c \phi(1)
\end{aligned}
$$

as desired. Now by definition we have

$$
\limsup F_{\beta}\left(\nu_{\beta}\right)=\limsup I_{\beta}+I I_{\beta},
$$

so it suffices to show that $I_{\beta} \rightarrow I$ and $I I_{\beta} \rightarrow I I$.

Now since $\int_{0}^{1} d \nu_{\beta} \rightarrow \int_{0}^{1} d \nu$, it follows that $\xi^{\prime \prime}(s) \int_{s}^{1} d \nu_{\beta}$ is bounded so that by the bounded convergence theorem

$$
\lim I_{\beta} \rightarrow \int \xi^{\prime \prime}(s) \nu[s, 1] d s
$$

Now consider

$$
I I_{\beta}=\int_{0}^{1} \frac{1}{\nu_{\beta}[s, 1]}\left(1-\frac{1}{\beta} \frac{\nu_{\beta}[s, 1]}{1-s}\right) d s=\int_{0}^{q_{\beta}} \frac{1}{\nu_{\beta}[s, 1]}\left(1-\frac{1}{\beta} \frac{\nu_{\beta}[s, 1]}{1-s}\right) d s .
$$

On $s \leq q_{\beta}$, we have

$$
\nu_{\beta}[s, 1]=\int_{s}^{q_{\beta}} m(t) d t+\beta\left(1-q_{\beta}\right)=\int_{s}^{q_{\beta}} m(t) d t+\int_{q_{\beta}}^{1} m(t) d t+c_{\beta}=\int_{s}^{1} m(t) d t+c_{\beta}
$$

so that

$$
I I_{\beta}=\int_{0}^{q_{\beta}} \frac{1}{\int_{s}^{1} m(t) d t+c_{\beta}}\left(1-\frac{1}{\beta} \frac{\int_{s}^{1} m(t) d t+c_{\beta}}{1-s}\right) d s
$$

Observe that

$$
0 \leq \frac{1}{\int_{s}^{1} m(t) d t+c_{\beta}}\left(1-\frac{1}{\beta} \frac{\int_{s}^{1} m(t) d t+c_{\beta}}{1-s}\right) \mathbb{1}_{\left[0, q_{\beta}\right]} \uparrow \frac{1}{\nu[s, 1]} \mathbb{1}_{[0,1)}
$$

so that by the monotone convergence theorem,

$$
I I_{\beta} \rightarrow \int_{0}^{1} \frac{1}{\nu[s, 1]} d s
$$

as desired. 


\subsubsection{Proof of Theorem 1.3.}

Proof. The $\Gamma$-convergence result for $h=0$ and $\xi_{\beta}=\xi$ follows by Lemma 2.1 and Lemma 2.2. In the case that, $\frac{h^{2}}{\beta^{2}} \rightarrow \bar{h}$ and $\xi_{\beta}^{\prime \prime} \rightarrow \xi^{\prime \prime}$ uniformly, the corresponding result then follows by a continuous perturbation argument (see Theorem 6.17).

We now turn to the convergence of minimizers and (1.1.3). First observe that by Lemma 6.3 . the family $F_{\beta}$ are sequentially equi-coercive. Since $G S$ has a unique minimizer, the result follows by Theorem 6.16 .

2.2. Moderate Deviations of the minimizers. In the following we study consequences of the $\Gamma$-convergence. In particular, we aim to prove Corollary 1.4. By Theorem 1.3 , we know that $\nu_{\beta}=\beta \mu_{\beta}[0, t] d t \rightarrow d \nu=m(t) d t+c \delta_{1}$ weakly where $d \nu$ is the unique minimizer of GS.

Lemma 2.4. We have $\beta \mu_{\beta}[0, t] \rightarrow m(t)$ for $t \in[0,1) \cap \operatorname{CtyPts}(m(t))$.

Proof. Let $f_{\beta}=\beta \mu_{\beta}[0, t]$. Observe first that since $f_{\beta} d t \rightarrow d \nu$, we have that for every $T \in[0,1)$,

$$
\sup _{t \in[0, T]}\left|f_{\beta}(t)\right|=f_{\beta}(T) \leq \frac{1}{1-T} \int_{T}^{1} f_{\beta}(t) d t=\frac{1}{1-T} \nu_{\beta}([T, 1]) \leq C(T) .
$$

Thus $f_{\beta}$, restricted to the interval $[0, T]$ is a sequence of uniformly bounded monotone functions. As a consequence, every subsequence has a further subsequence that converges point-wise on $[0, T]$ to some function $f(t)$, and that $f(t)$ also has this bound.

But then, by the dominated convergence theorem applied to this subsequence,

$$
\int_{0}^{T} g(t) f_{\beta}(t) d t \rightarrow \int_{0}^{T} g(t) f(t) d t
$$

for any $g \in L^{1}[0, T]$. As a result, $f(t)=m(t)$ a.e. on $[0, T]$. By monotonicity, $f(t)=m(t)$ at their continuity points on $[0, T]$. The subsequence principle applied to $f_{\beta}(t)$ at continuity points of $m(t)$ on $[0, T]$. Since this holds for each $T<1$ we conclude the result.

Lemma 2.5. Let $q_{\beta} \rightarrow 1$ be such that $\beta \mu_{\beta}\left[0, q_{\beta}\right) \rightarrow m\left(1^{-}\right)$, and suppose that $m\left(1^{-}\right)<\infty$. If $Y_{\beta}$ have law $\mu_{\beta}$, then

$$
\nu(\{1\})=\lim \mathbb{E}_{\mu_{\beta}}\left(\beta\left(1-Y_{\beta}\right) \mid Y_{\beta} \in\left[q_{\beta}, 1\right]\right) .
$$

Proof. Note that since $m\left(1^{-}\right)<\infty$ and $\mu_{\beta}[0,1]=1$, so that $\mu\left[q_{\beta}, 1\right] \rightarrow 1$. Thus it suffices to show that

To see this observe that

$$
\lim \beta \int \mathbb{1}_{\left[q_{\beta}, 1\right]}(1-t) d \mu_{\beta} \rightarrow \nu(\{1\}) .
$$

$$
\beta \int_{0}^{1} \mu_{\beta}[0, t] d t=\beta \int_{0}^{q_{\beta}} \mu_{\beta}[0, t] d t+\beta \mu\left[0, q_{\beta}\right)\left(1-q_{\beta}\right)+\beta \int_{q_{\beta}}^{1} \mu_{\beta}\left[q_{\beta}, t\right] d t=I+I I+I I I .
$$

Observe that $I I \rightarrow 0$ by assumption. Furthermore observe that

$$
I I I=\beta \int \mathbb{1}_{\left[q_{\beta}, 1\right]}(1-t) d \mu_{\beta} .
$$

Since $\nu=m(t) d t+c \delta_{1}$ and $\nu_{\beta} \rightarrow \nu$,

$$
\beta \int_{0}^{1} \mu_{\beta}[0, t] d t \rightarrow \int_{0}^{1} m(t) d t+c
$$

It thus suffices to show that $I \rightarrow \int_{0}^{1} m(t) d t$. To see this observe that if we define $g_{\beta}(t)=$ $\mathbb{1}_{\left[0, q_{\beta}\right]} \beta \mu_{\beta}[0, t]$, then

$$
\sup \left|g_{\beta}(t)\right| \leq \underset{14}{\beta \mu_{\beta}}\left[0, q_{\beta}\right) \leq C
$$


and $g_{\beta}(t) \rightarrow m(t)$ for $\mathcal{L}$-a.e. $\mathrm{t}$ by Lemma 2.4 . The result then follows by the dominated convergence theorem.

2.2.1. Proof of Corollary 1.4.

Proof. Proof of (1). Theorem 1.3 implies $\beta \mu_{\beta}[0, t] d t \rightarrow d \nu$. A simple integration by parts argument then shows the first result.

Proof of (2). The second result follows from Lemma 2.4 .

Proof of (3). The third result comes from Lemma 2.5.

\section{The Dual of the ground state energy Problem}

In this section, we prove Theorem 1.10. We follow the usual method of introducing an auxiliary function and proving a minmax theorm for it. The result then follows by studying the min-max and the max-min problems. We then present a preliminary analysis of the two functionals which will be important for the regularity theory of these problems. For the purposes of this section, we will think of $\xi$ and $h$ as fixed and write $P=P_{\xi, h}$ when it is unambiguous.

3.1. Proof of duality. In this subsection we prove the duality theorem, Theorem 1.10. We introduce the following notation for ease of comparison with [4, 22]. This notation, with the exception of the set $B$, will be used only for the following lemma. Let $F=C([0,1]), G=\mathscr{M}([0,1])$, $A=\mathcal{C}$, and

$$
B=\left\{\mu \in \mathscr{M}_{+}: \mu(\{0,1\})=0\right\} .
$$

Let $S(\nu): \mathscr{M}_{+} \rightarrow \mathbb{R}$ be defined by

$$
S(\nu)=2 \int \sqrt{\nu_{a c}(x)} d x .
$$

Note that by Jensen's inequality this is finite. Basic regularity of $S$ is shown in Section 6.3 . One important fact from the latter section that will be used frequently in the subsequent is the representation

$$
S(\nu)=\inf _{\phi \geq 0} \int \phi d \nu+\int \frac{1}{\phi} d x
$$

Define $K: A \times B \rightarrow \mathbb{R}$ by

$$
K(u, v)=\left(\xi^{\prime \prime}-v, u\right)+S(v)+h^{2} u(0) .
$$

Give $F$ the norm topology and $G$ the norm-topology, and give $A, B$ the induced topologies.

Lemma 3.1. We have

$$
\inf _{u \in A} \sup _{v \in B} K(u, v)=\sup _{v \in B} \inf _{u \in A} K(u, v) .
$$

Proof. We use a generalization of Ky Fan's min-max theorem due to Brezis, Nirenberg, Stampacchia [4, 22]. Note that $A, B$ are convex sets, and that $F$ is a Hausdorff topological vector space. We need to check

(1) For each $v \in B, K$ is quasi-convex in $u$ and l.s.c. on $A$

(2) For each $u \in A, K$ is quasi-concave in $v$ and u.s.c. on $B$

(3) For some $\tilde{v} \in B$, and some $\lambda>\sup _{B} \inf _{A} K$, the set $\{u \in A: K(u, \tilde{v}) \leq \lambda\}$ is compact.

Then the Generalized Ky Fan min-max theorem will imply the result.

Pf of 1 . Let $v \in B$, then the map

$$
A \rightarrow \mathbb{R}, u \rightarrow K(u, v)
$$

is affine with continuous linear part, hence it is (quasi-)convex and continuous. 
Pf of 2. Let $u \in A$, then the map

$$
B \rightarrow \mathbb{R}, v \rightarrow K(u, v)
$$

is concave by concavity of $x \mapsto \sqrt{x}$. It is upper semi-continuous by Corollary 6.14 .

Pf of 3. We begin by asserting that $\sup _{B} \inf _{A} K<\infty$. To see that, let $P$ is as in 1.2 .2 . By 3.1.1, it follows for every $u \in A$,

$$
\sup _{v \in B} K(u, v) \leq P(u)
$$

so that

$$
\sup _{B} \inf _{A} K \leq \inf _{A} \sup _{B} K \leq \inf _{A} P \leq P(1)<\infty
$$

Therefore there exists $\lambda \in \mathbb{R}$ such that $\lambda>\sup _{B} \inf _{A} K$. Since the set

$$
E=\{u \in A: K(u, 0) \leq \lambda\}
$$

is a closed subset of the set

$$
\left\{u \in A: 0 \leq\left(\xi^{\prime \prime}, u\right) \leq \lambda\right\}
$$

Lemma 6.6 in the case $f=\xi^{\prime \prime}$, yields that $E$ is norm compact in $C[0,1]$.

Theorem 3.2. We have

$$
\min _{\phi \in \mathcal{C}} P(\phi)=\sup _{\eta \in \mathcal{K}_{h, \xi}} D(\eta) .
$$

Proof. Our first goal is to show that for all $\phi \in A$,

$$
\sup _{\sigma \in B} K(\phi, \sigma)=P(\phi) .
$$

The upper bound is given by 3.1 .2 . On the other hand, for all $\epsilon>0$ we let $\sigma_{\epsilon}(d x)=\frac{1}{(\phi+\epsilon)^{2}} d x$, to get that

$$
\begin{aligned}
\inf _{\sigma \in B}\left((\phi, \sigma)-\int 2 \sqrt{\sigma_{a c}(x)} d x\right) & \leq \int \frac{\phi}{(\phi+\epsilon)^{2}} d x-2 \int \frac{1}{(\phi+\epsilon)} d x \\
& \leq-\int \frac{1}{\phi+\epsilon} d x \rightarrow-\int \frac{1}{\phi} d x
\end{aligned}
$$

as $\epsilon \rightarrow 0$ by the monotone convergence theorem. Thus 3.1 .3 holds.

Next we show that for all $\sigma \in B$,

$$
\inf _{\phi \in \mathcal{C}} K(\phi, \sigma)=S(\sigma)+\Xi(\sigma)
$$

where

$$
\Xi(\sigma)=\inf _{\phi \in \mathcal{C}}\left(\left(\phi, \xi^{\prime \prime}\right)-(\phi, \sigma)+h^{2} \phi(0)\right)= \begin{cases}0 & \sigma=\eta^{\prime \prime}, \eta \in \mathcal{K}_{h, \xi} \\ -\infty & \text { otherwise }\end{cases}
$$

The first equality is self-evident. The issue is to show the second equality.

Suppose first that there is no $\eta \in K_{\xi}$ such that $\eta^{\prime \prime}=\sigma$. Define $\eta$ to be the solution of

$$
\left\{\begin{array}{l}
\eta^{\prime \prime}=\sigma \\
\eta^{\prime}(0)=\xi^{\prime}(0)-h^{2} \\
\eta(1)=\xi(1)
\end{array}\right.
$$

(see Lemma 6.10). Observe that $\eta$ is continuous, convex, and has the boundary data from the definition of $\mathcal{K}_{h, \xi}$. Thus it must be that $\{\xi>\eta\}$ is non-empty. 
Take $L \in \mathbb{R}_{+}$and $\phi$ satisfying $\phi^{\prime}(0)=0, \phi(1)=0$, and $-\phi^{\prime \prime}=L \mu$ where $\mu$ is a probability measure supported in a compact subset of $\{\xi>\eta\} \backslash\{0,1\}$. Since $\phi \in \mathcal{C} \cap\left\{\phi^{\prime} \in B V\right\}$ it follows by Lemma 6.11 that

$$
\begin{aligned}
\left((\xi-\eta)^{\prime \prime}, \phi\right)+h^{2} \phi(0) & =\left.(\xi-\eta)^{\prime} \phi\right|_{0} ^{1}-\left.(\xi-\eta) \phi^{\prime}\right|_{0} ^{1}+\left(\eta-\xi,-\phi^{\prime \prime}\right)+h^{2} \phi(0) \\
& =L(\eta-\xi, \mu) .
\end{aligned}
$$

Taking $L \rightarrow \infty$ gives the result.

We now need to show that the infimum is 0 when $\sigma=\eta^{\prime \prime}$ for some $\eta \in \mathcal{K}_{h, \xi}$. To see this note that by definition of $\mathcal{K}_{h, \xi}$, if $\phi^{\prime}(1)>-\infty$, the same integration by parts argument yields

$$
\left((\xi-\eta)^{\prime \prime}, \phi\right)+h^{2} \phi(0)=(\xi-\eta)^{\prime}(1) \phi(1)+(\xi-\eta)(0) \phi^{\prime}(0)+\left(\eta-\xi,-\phi^{\prime \prime}\right) \geq 0 .
$$

We used here that $\eta \in \mathcal{K}_{h, \xi}$ then $(\xi-\eta)^{\prime}(1) \geq 0$ by Lemma 6.9. Now if $\phi^{\prime}(1)=-\infty$, we take a sequence of $\phi_{n} \in \mathcal{C}$ with $\phi_{n} \rightarrow \phi$ in norm with $\phi_{n}^{\prime}(1)>-\infty$ (see Lemma 6.8), for which the inequality still holds and then pass to the limit.

The duality then follows by Lemma 3.1 which implies that

$$
\inf _{\phi \in \mathcal{C}} P(\phi)=\inf _{\phi \in A} \sup _{\sigma \in B} K(\phi, \sigma)=\sup _{\sigma \in B} \inf _{\phi \in A} K(\phi, \sigma)=\sup _{\eta \in \mathcal{K}_{\xi}} D\left(\eta^{\prime \prime}\right) .
$$

3.2. Preliminary Analysis of the Primal-Dual Relationship. In this section we do some preliminary analyses of the Primal and Dual problems and their relationship which will be used in the subsequent.

Lemma 3.3. $(\phi, \eta) \in \mathcal{C} \times \mathcal{K}_{h, \xi}$. The following are equivalent:

- $P(\phi)=D(\eta)$

- $\phi^{2} \eta^{\prime \prime}(d x)=d x$ and $\int \phi \xi^{\prime \prime}+h^{2} \phi(0)=\int \phi \eta^{\prime \prime}$

- $\phi$ and $\eta$ optimize $P$ and $D$ respectively.

Furthermore, if $P(\phi)=D(\eta)$, we have that $\frac{1}{\phi^{2}} \in L^{1}, \eta^{\prime \prime}<<d x, \eta^{\prime \prime}(d x)=\frac{1}{\phi^{2}} d x$, and

$$
\begin{gathered}
\xi^{\prime}(1)=\eta^{\prime}(1) \text { or } \phi(1)=0 \\
\xi(0)=\eta(0) \text { or } \phi^{\prime}(0)=0 .
\end{gathered}
$$

Proof. By the same argument as in Theorem 3.2, for any such pair we have the inequality

$$
I=\left(\phi, \xi^{\prime \prime}\right)-\left(\phi, \eta^{\prime \prime}\right)+h^{2} \phi(0) \geq 0 .
$$

In fact, any such pair must satisfy

$$
I I=\int \frac{1}{\phi}+\left(\phi, \eta^{\prime \prime}\right)-S\left(\eta^{\prime \prime}\right) \geq 0 .
$$

To see this, observe that

$$
I I=\int \frac{1}{\phi(x)}+\phi(x) \eta_{a c}^{\prime \prime}(x)-2 \sqrt{\eta_{a c}^{\prime \prime}(x)} d x+\int \phi \eta_{s i n g}^{\prime \prime}(d x)
$$

which is non-negative as both integrands are non-negative. Hence,

$$
P(\phi)-D(\eta)=I+I I \geq 0
$$

with the case of equality if and only if $I=I I=0$.

From the statement that $I=0$, we conclude by an integration by parts and approximation argument (as in the proof of Theorem 3.2 ) that

$$
\begin{gathered}
\xi^{\prime}(1)=\eta^{\prime}(1) \text { or } \phi(1)=0 \\
\xi(0)=\eta(0) \text { or } \phi^{\prime}(0)=0 .
\end{gathered}
$$


Furthermore, we have that $I I=0$ if and only if $\phi^{2} \eta^{\prime \prime}(d x)=d x$. Indeed, if $I I=0$, then $\phi^{2}(x) \eta_{a c}^{\prime \prime}(x)=1 \mathcal{L}$-a.e. and $\operatorname{supp} \eta_{\text {sing }}^{\prime \prime} \subset\{\phi=0\}$. Since $\frac{1}{\phi} \in L^{1}$, it follows from monotonicity of $\phi$ that $\eta_{\text {sing }}^{\prime \prime}(d x)=0$. Hence $\phi^{2} \eta^{\prime \prime}(d x)=d x$ and $\eta_{a c}^{\prime \prime}=\frac{1}{\phi^{2}} \in L^{1}$. The reverse direction is clear.

That $P(\phi)=D(\eta)$ if and only if $\phi$ and $\eta$ are optimal is an immediate consequence of (3.2.1).

Lemma 3.4. Let $\phi \in \mathcal{C}$ then for any $\psi \in \mathcal{C}$,

$$
\left.\frac{d}{d \tau}\right|_{\tau=0^{+}} P(\phi+\tau \psi)=\left(\xi^{\prime \prime}-\frac{1}{\phi^{2}}, \psi\right)+h^{2} \psi(0)
$$

Furthermore, $\phi$ is optimal only if

$$
\left(\xi^{\prime \prime}-\frac{1}{\phi^{2}}, \psi\right)+h^{2} \psi(0) \geq 0 .
$$

Proof. Notice that it suffices to show that the nonlinear term is right differentiable. Since $\psi \in \mathcal{C}$, so is $\phi_{\tau}=\phi+\tau \psi$. Now $\psi \geq 0$ so that $1 / \phi_{\tau}$ is a non-negative, monotone increasing sequence of functions. then

$$
\lim _{\tau \rightarrow 0^{+}}-\frac{1}{\tau} \int \frac{1}{\phi_{\tau}}-\frac{1}{\phi} d x=\lim _{\tau \rightarrow 0^{+}} \int \frac{\psi}{\phi_{\tau} \phi} d x=\int \frac{1}{\phi^{2}} \psi d x
$$

by the monotone convergence theorem so that the non-linear term in $P$ is right differentiable at $\tau=0$. The second claim follows from first order optimality.

Lemma 3.5. If $\phi$ optimizes $P(\cdot)$, then $\frac{1}{\phi^{2}} \in L_{1}$. In particular,

$$
\eta(x)=\xi(1)-\int_{x}^{1} \int_{0}^{y} \frac{1}{\phi^{2}} d z d y+h^{2}(1-t)
$$

is in $\mathcal{K}_{h, \xi} \cap W^{2,1}$.

Proof. Fix $\nu \in \mathscr{M}_{+}$and let $\psi(t)=a+c(1-t)+\int_{t}^{1} \nu[0, s] d t$ then $\psi \in \mathcal{C}$ so that by Lemma 3.4 .

$$
\left.\frac{d}{d \tau}\right|_{\tau=0} P(\phi+\tau \psi)=\int\left(\xi^{\prime \prime}-\frac{1}{\phi^{2}}\right) \psi d t+h^{2} a \geq 0
$$

Choosing $a=1, c=0$, and $\nu=0$ gives

$$
\int \xi^{\prime \prime}-\frac{1}{\phi^{2}} d t+h^{2} \geq 0
$$

so that

$$
h^{2}+\int \xi^{\prime \prime} \geq \int \frac{1}{\phi^{2}} \geq 0 .
$$

(To avoid adding infinities, subtract $h^{2}+\int \xi^{\prime \prime}$ from both sides and use the a priori sign on $-\frac{1}{\phi^{2}}$.)

$\eta(x)$ is plainly continuous, convex, and has the correct boundary data. It suffices to show that $\eta \geq \xi$. Observe that for any $\psi \in \mathcal{C}$ with $\psi^{\prime}(1)>-\infty$, we have

$$
0 \leq\left(\psi, \xi^{\prime \prime}-\frac{1}{\phi^{2}}\right)+h^{2} \psi(0)=\psi(1)\left(\xi^{\prime}-\eta^{\prime}\right)(1)+\psi^{\prime}(0)(\xi-\eta)(0)+\left(\psi^{\prime \prime}, \xi-\eta\right)
$$

by Lemma 3.4 . Taking $\psi$ with $\psi(1)=0, \psi^{\prime}(0)=0$ and $\psi^{\prime \prime}=-\delta_{t}$ for $t \in(0,1)$ shows that $\eta(t) \geq \xi(t)$. Then inequality then extends by continuity. Thus $\eta \in \mathcal{K}_{h, \xi}$. That $\eta \in W^{2,1}$ is immediate.

Theorem 3.6. (Well-posedness) The Dual problem is well-posed. In particular,

$$
\sup _{\eta \in \mathcal{K}_{h, \xi}} D(\eta)=\max _{\eta \in \mathcal{K}_{h, \xi}} D(\eta) \text {. }
$$

Furthermore, $\eta$ is unique.

Proof. This from the fact that $P$ has a unique minimizer combined with Lemma 3.3 and Lemma 3.5. 


\section{REgUlARITY THEORY FOR THE OPTIMIZERS}

In this section we prove Theorem 1.11 and Theorem 1.13 . In this section, $\phi$ and $\eta$ refer exclusively to the optimizers. (That there exists a unique optimizer $\eta$ was proved in the previous section.)

4.1. Primal and Dual Regularity. Our goal in this section is to prove Theorem 1.11. We will prove this by first getting weaker regularity using the above and then we will upgrade this regularity.

Notice that by Lemma 3.3, we have the following lemma.

Lemma 4.1. We have that

(1) $\phi>0$ on $[0,1)$

(2) $\eta \in C_{l o c}^{2}((0,1)) \cap C^{1}([0,1])$.

(3) $\frac{1}{\sqrt{\eta^{\prime \prime}}}=\phi$ on $(0,1)$

(4) on the set $\{\xi=\eta\} \cap(0,1), \eta^{\prime}(t)=\xi^{\prime}(t)$, and $\eta^{\prime \prime}(t) \geq \xi^{\prime \prime}(t)$.

Proof. Begin by observing that (1) - (3) follow from Lemma 3.3 and monotonicity of $\phi$.

To see $(4)$, fix $t \in(0,1)$ a contact point. Begin by recalling that $\eta$ is left/right differentiable and lies above $\xi$ by definition of $\mathcal{K}$, so $\xi^{\prime}(t)$ is in the sub-differential

$$
\partial_{-} \eta(t) \leq \xi^{\prime}(t) \leq \partial_{+} \eta(t)
$$

but $\eta \in C_{l o c}^{2}((0,1))$ so these are in fact equalities. Furthermore if we define $g=\eta-\xi \geq 0$, then for $\epsilon$ sufficiently small,

$$
g(t+\epsilon)=g(t)+g^{\prime}(t) \epsilon+\frac{g^{\prime \prime}(\tau)}{2} \epsilon^{2}=\frac{g^{\prime \prime}(\tau)}{2} \epsilon^{2}
$$

for some $\tau$ between $t$ and $t+\epsilon$ where the second equality follows by the observations follows by the argument earlier in the lemma. Since $g \in C_{l o c}^{2}((0,1))$ and $g \geq 0$ we conclude that $g^{\prime \prime}(t) \geq 0$ by taking $\epsilon \rightarrow 0$.

Lemma 4.2. We have that $\left(\frac{1}{\sqrt{\eta^{\prime \prime}}}\right)^{\prime \prime}=-\mu$ in the sense of $\mathcal{D}^{\prime}$ where $\mu$ is a positive Radon measure on $(0,1)$ which has support in $\{\xi=\eta\}$.

Proof. By Lemma $4.1, \frac{1}{\sqrt{\eta^{\prime \prime}}}$ is concave so that the statement

$$
\left(\frac{1}{\sqrt{\eta^{\prime \prime}}}\right)^{\prime \prime}=-\mu
$$

follows from Alexandrov's theorem (Theorem 6.12). To find the condition on the support, it suffices to consider the case that the contact set is not the whole interval. Then there is an $\epsilon_{0}>0$ and an open set $U$ with $U \subset \subset\left\{\eta-\xi>\epsilon_{0}\right\}$. Let $\sigma$ be a bump function that is localized in $U$. Note that we can take $U$ to be away from $\{0,1\}$. Now $\eta \in C_{l o c}^{2}(0,1)$ by Lemma 4.1 so that it is $C^{2}(\bar{U})$. Furthermore $\eta^{\prime \prime}(x) \geq \inf _{U} \frac{1}{\phi^{2}}>0$. Thus one can directly verify that $\eta+\tau \sigma \in K_{\xi}$ for $|\tau|$ sufficiently small. This implies

$$
\left.\frac{d}{d \tau}\right|_{\tau=0} S(\eta+\tau \sigma)=\left(\frac{1}{\sqrt{\eta^{\prime \prime}}}, \sigma^{\prime \prime}\right)=0
$$

by the optimality of $\eta$ and the fact that this is a full derivative (i.e. not just a right or left derivative). The result then follows by the definition of distributional derivatives.

Lemma 4.3. There is a $c>0$ with $0<c \leq \phi$. In particular $\eta \in C^{2}([0,1])$, and the second derivative has the strict lower bound $\eta^{\prime \prime}(x)>c^{-2}$. 
Proof. We do a case analysis. Suppose there exists a sequence of interior contact points $\left\{t_{n}\right\}$ with $t_{n} \uparrow 1$. Then by Lemma 4.1, you have that $\eta^{\prime}\left(t_{n}\right)=\xi^{\prime}\left(t_{n}\right)$ for all $n$, so then by mean value theorem there exists a sequence of points $\left\{\tilde{t}_{n}\right\}$ with $\tilde{t}_{n} \uparrow 1$ at which $\eta^{\prime \prime}\left(\tilde{t}_{n}\right)=\xi^{\prime \prime}\left(\tilde{t}_{n}\right)$. Then by conjugacy you have that $\phi^{2}\left(\tilde{t}_{n}\right)=\frac{1}{\sqrt{\xi^{\prime \prime}\left(\tilde{t}_{n}\right)}}$ and therefore $\phi^{2}(1)=\frac{1}{\sqrt{\xi^{\prime \prime}(1)}}$ which gives a lower bound on $\phi$.

On the other hand, if no such sequence of contact points exists, then there is an interval of the form $(a, 1) \subset\{\xi<\eta\}$. By Lemma $4.2,\left(\frac{1}{\sqrt{\eta^{\prime \prime}}}\right)^{\prime \prime}=0$ in the sense of distributions on $(a, 1)$. By Lemma 4.1 and elementary properties of distributions [19], it follows that $\phi$ is linear on $(a, 1)$. Combined with finite energy you conclude that $\phi(1) \neq 0$. This gives a lower bound on $\phi$ by monotonicity. That $\eta \in C^{2}$ then follows by a continuity argument.

Lemma 4.4. We have that $\phi^{\prime}(1)>-\infty$. Thus, $\phi \in C([0,1]) \cap\left\{\phi^{\prime} \in B V((0,1))\right\}$. For the corresponding $d \nu=m(t) d t+c \delta_{1} \in \mathcal{A}$, we have that $m(1-)<\infty$.

Proof. Note that by Lemma 4.1 and Lemma 4.3 , we have that $\eta^{\prime \prime}(x)=\frac{1}{\phi^{2}(x)}$ for all $x \in[0,1]$. Thus, $\eta^{\prime \prime}$ is left-differentiable at 1 (with possibly infinite value) and in particular,

$$
\begin{aligned}
\eta^{\prime \prime \prime}(1) & =\lim _{t \rightarrow 1^{-}} \frac{\eta^{\prime \prime}(1)-\eta^{\prime \prime}(t)}{1-t}=\lim _{t \rightarrow 1^{-}} \frac{\frac{1}{\phi^{2}(1)}-\frac{1}{\phi^{2}(t)}}{1-t} \\
& =\lim _{t \rightarrow 1^{-}} \frac{\phi^{2}(t)-\phi^{2}(1)}{(1-t) \phi^{2}(1) \phi^{2}(t)}=\lim _{t \rightarrow 1^{-}} \frac{(\phi(t)-\phi(1))}{(1-t)} \frac{(\phi(t)+\phi(1))}{\phi^{2}(1) \phi^{2}(t)} \\
& =-\phi^{\prime}(1) \frac{2}{\phi^{3}(1)} .
\end{aligned}
$$

Since $\phi^{3}(1) \in(0, \infty)$, we see that the first claim is equivalent to proving that $\eta^{\prime \prime \prime}(1)<\infty$.

Now we proceed by a case analysis. Suppose first that 1 is an isolated contact point. Then as in the proof of Lemma 4.3 we conclude that $\phi$ is linear on an interval of the form $(1-\delta, 1)$. It follows immediately that $\phi^{\prime}(1)>-\infty$.

Suppose now that 1 is not an isolated contact point. Then there is a monotone sequence $t_{i} \in$ $(0,1)$ such that $t_{i} \uparrow 1$ and $\eta\left(t_{i}\right)=\xi\left(t_{i}\right)$. By Lemma 4.1. we have that $\eta^{\prime}\left(t_{i}\right)=\xi^{\prime}\left(t_{i}\right)$ and that $\eta^{\prime \prime}\left(t_{i}\right) \geq \xi^{\prime \prime}\left(t_{i}\right)$. This easily implies that $\eta^{\prime \prime \prime}(1)=\xi^{\prime \prime \prime}(1)<\infty$. To see this, observe that by the mean value theorem there is a sequence $\tau_{i} \in\left(t_{i}, t_{i+1}\right)$ such that $\eta^{\prime \prime}\left(\tau_{i}\right)=\xi^{\prime \prime}\left(\tau_{i}\right)$. Then, $\eta^{\prime \prime}(1)=\xi^{\prime \prime}(1)$ since $\eta \in C^{2}([0,1])$. Hence,

$$
\begin{aligned}
\eta^{\prime \prime \prime}(1) & =\lim _{t \rightarrow 1} \frac{\eta^{\prime \prime}(1)-\eta^{\prime \prime}(t)}{1-t}=\lim _{i \rightarrow \infty} \frac{\eta^{\prime \prime}(1)-\eta^{\prime \prime}\left(\tau_{i}\right)}{1-\tau_{i}} \\
& =\lim _{i \rightarrow \infty} \frac{\xi^{\prime \prime}(1)-\xi^{\prime \prime}\left(\tau_{i}\right)}{1-\tau_{i}}=\xi^{\prime \prime \prime}(1)
\end{aligned}
$$

as required.

Having shown that $\phi^{\prime}(1)>-\infty$, we immediately conclude that $\phi^{\prime} \in B V$ (see Lemma 6.7). The last claim follows from the correspondence between $\mathcal{A}$ and $\mathcal{C}$, which gives that $\phi(t)=\int_{t}^{1} m(t)+c$ for all $t \in[0,1]$ and hence that $m\left(1^{-}\right)=-\phi^{\prime}(1)<\infty$.

\subsubsection{Proof of Theorem 1.11.}

Proof. This follows from applying Lemmas 3.3, 4.1, 4.2, 4.3, and 4.4.

4.2. Regularity of the contact set. In this section we prove Theorem 1.13 . This result, which we restate below for the convenience of the reader, provides a simple test which characterizes which sub-intervals of $[0,1]$ are compatible with FRSB and which are compatible with $k R S B$. 
Theorem. 1.13. Let

$$
\mathfrak{d}(t)=\left(\frac{1}{\sqrt{\xi^{\prime \prime}}}\right)^{\prime \prime}(t)
$$

Then we have the following two cases

(1) If $\mathfrak{d}(t)>0$ on $(a, b) \subset[0,1]$, then there are at most two contact points in $[a, b]$.

(2) If $\mathfrak{d}(t) \leq 0$ on $[a, b] \subset(0,1]$, then if there are two contact points $t_{1}, t_{2} \in[a, b]$, then $\left[t_{1}, t_{2}\right] \subset$ $\{\eta=\xi\}$.

Proof. We begin with 1. Let $\eta$ be optimal for the dual problem, and suppose that $\mathfrak{d}(t)>0$ on $[a, b]$. By contradiction, assume there are three contact points at $a \leq t_{1}<t_{2}<t_{3} \leq b$. Note that by Theorem 1.11. $\eta^{\prime}\left(t_{i}\right)=\xi^{\prime}\left(t_{i}\right)$ for $i=1,2,3$, and $\eta^{\prime \prime}\left(t_{2}\right) \geq \xi^{\prime \prime}\left(t_{2}\right)$. By the mean value theorem, there exist points $s_{1}, s_{2}$ with $t_{1}<s_{1}<t_{2}<s_{2}<t_{3}$ such that $\eta^{\prime \prime}\left(s_{i}\right)=\xi^{\prime \prime}\left(s_{i}\right)$ for $i=1,2$. By Theorem 1.11, $t \rightarrow \frac{1}{\sqrt{\eta^{\prime \prime}(t)}}$ is concave, and hence the function $t \rightarrow \frac{1}{\sqrt{\eta^{\prime \prime}(t)}}-\frac{1}{\sqrt{\xi^{\prime \prime}(t)}}$ is strictly concave on $(a, b)$ by the assumption on $\mathfrak{d}$. As this function vanishes at the points $s_{1}, s_{2}$, it must be strictly positive between. In particular, you have that $\xi^{\prime \prime}\left(t_{2}\right)>\eta^{\prime \prime}\left(t_{2}\right)$, which is a contradiction.

Now we turn to 2 . Suppose now that $\mathfrak{d} \leq 0$ on $[a, b] \subset(0,1]$. We claim that

$$
\int_{t_{1}}^{t_{2}}\left(\frac{1}{\sqrt{\xi^{\prime \prime}}}-\frac{1}{\sqrt{\eta^{\prime \prime}}}\right)(\xi-\eta)^{\prime \prime} \geq 0
$$

With this claim in hand, observe that

$$
\frac{1}{\sqrt{\xi^{\prime \prime}}}-\frac{1}{\sqrt{\eta^{\prime \prime}}}=\frac{1}{\sqrt{\xi^{\prime \prime} \cdot \eta^{\prime \prime}}\left(\sqrt{\eta^{\prime \prime}}+\sqrt{\xi^{\prime \prime}}\right)}\left(\eta^{\prime \prime}-\xi^{\prime \prime}\right)
$$

so that the claim implies that

$$
\int_{t_{1}}^{t_{2}} \frac{1}{\sqrt{\xi^{\prime \prime} \cdot \eta^{\prime \prime}}\left(\sqrt{\eta^{\prime \prime}}+\sqrt{\xi^{\prime \prime}}\right)}\left(\xi^{\prime \prime}-\eta^{\prime \prime}\right)^{2} \leq 0
$$

The integrand, however, is nonnegative by the same argument. This implies that in fact

$$
\frac{1}{\sqrt{\xi^{\prime \prime} \cdot \eta^{\prime \prime}}\left(\sqrt{\eta^{\prime \prime}}+\sqrt{\xi^{\prime \prime}}\right)}\left(\xi^{\prime \prime}-\eta^{\prime \prime}\right)^{2}=0
$$

for $\mathcal{L}$-a.e. point in $\left[t_{1}, t_{2}\right]$, and hence $\eta^{\prime \prime}=\xi^{\prime \prime}$ for $\mathcal{L}$-a.e. point in $\left[t_{1}, t_{2}\right]$. Since $\eta=\xi$ at $t_{1}, t_{2}$ we conclude the result.

Now we prove the claim. First, note that by the assumption on $\mathfrak{d}$,

$$
\left(\frac{1}{\sqrt{\xi^{\prime \prime}}}\right)^{\prime \prime}(\eta-\xi) \leq 0 \quad t \in\left[t_{1}, t_{2}\right]
$$

since $\eta \in \mathcal{K}_{h, \xi}$, so that

$$
\int_{t_{1}}^{t_{2}}\left(\frac{1}{\sqrt{\xi^{\prime \prime}}}\right)^{\prime \prime}(\eta-\xi) \leq 0 .
$$

Integrate by parts and use the boundary conditions given by contact at $t_{1}, t_{2}$ to find that

$$
\int_{t_{1}}^{t_{2}} \frac{1}{\sqrt{\xi^{\prime \prime}}}(\eta-\xi)^{\prime \prime} \leq 0
$$

On the other hand, define

$$
\tilde{\eta}(t)= \begin{cases}\eta(t) & t \notin\left[t_{1}, t_{2}\right] \\ \xi(t) & t \in\left[t_{1}, t_{2}\right]\end{cases}
$$

and note that $\tilde{\eta} \in \mathcal{K}_{h, \xi}$. Furthermore, integration by parts and Theorem 1.11 , yield $\tilde{\eta}^{\prime \prime} \in L^{1}$ with the obvious expression. 
A first variation calculation and optimality of $\eta$ then yields the first order optimality condition

$$
\int_{t_{1}}^{t_{2}} \frac{1}{\sqrt{\eta^{\prime \prime}}}(\xi-\eta)^{\prime \prime} \leq 0
$$

Subtracting this from the above gives the claim that

$$
\int_{t_{1}}^{t_{2}}\left(\frac{1}{\sqrt{\xi^{\prime \prime}}}-\frac{1}{\sqrt{\eta^{\prime \prime}}}\right)(\xi-\eta)^{\prime \prime} \geq 0
$$

\section{5. $1 \mathrm{RSB}$}

In this section, we will study the special case that the minimizer is $1 \mathrm{RSB}$. We begin first with a study of this in the abstract. We remind the reader of the terminology from Section 1.3.2, and in particular the modified definition of $R S B_{1}$ given by $(1.3 .2)$.

5.1. 1RSB. In this subsection, we will prove Corollary 1.23. Recall the notion of formal conjugate from Section 1.3.2. Observe that the $\eta$ given by 1.3.4 in that section is continuous, convex, and satisfies the correct boundary data for $\mathcal{K}_{\xi}$. However, $\eta$ does not necessarily satisfy the obstacle condition.

Regarding the natural boundary conditions in Theorem 1.11, we have the following result whose proof is a straightforward calculation.

Fact 5.1. Let $\phi \in R S B_{1}$ and let $R$ be given by (1.3.5). Then we have that

$$
\begin{aligned}
R(t) & =\frac{1}{m}\left(\frac{1}{m} \log \left(\frac{c+m(1-t)}{c}\right)-\frac{1-t}{c+m}\right) \\
R^{\prime}(t) & =\frac{1}{m}\left(\frac{1}{c+m}-\frac{1}{c+m(1-t)}\right) .
\end{aligned}
$$

Furthermore, the formal conjugate $\eta$ to $\phi$ satisfies

$$
\left\{\begin{array}{l}
\eta(0)=\xi(0) \\
\eta^{\prime}(1)=\xi^{\prime}(1)
\end{array}\right.
$$

if and only if $m$ and $c$ solve

$$
\left\{\begin{array}{l}
\xi(1)=\frac{1}{m}\left(\frac{1}{m} \log \left(\frac{c+m}{c}\right)-\frac{1}{c+m}\right) \\
\frac{1}{\xi^{\prime}(1)}=c(c+m)
\end{array} .\right.
$$

The system (5.1.1) can be simplified by eliminating $m$ for $c$. That one can solve this system is proved in Fact 5.5 .

Lemma 5.2. Let $m, c>0$ solve (5.1.1). Then $c^{2} \xi^{\prime}(1)<1$, and $c$ solves the master equation

$$
\frac{\xi(1)}{\xi^{\prime}(1)}=\frac{c^{2} \xi^{\prime}(1)}{1-c^{2} \xi^{\prime}(1)}\left(\frac{1}{1-c^{2} \xi^{\prime}(1)} \log \left(\frac{1}{c^{2} \xi^{\prime}(1)}\right)-1\right) .
$$

Proof. Since $m, c$ are positive, we have that

$$
\frac{1}{\xi^{\prime}(1)}=c^{2}\left(1+\frac{m}{c}\right)>c^{2} .
$$

Showing that $c$ solves the master equation is a manipulation. Rewrite the first equation as

$$
c\left(\frac{1}{c^{2} \xi^{\prime}(1)}-1\right)=m,
$$


then plug this into (5.1.1) to find that

$$
\begin{aligned}
\xi(1) & =\left(\frac{1}{c\left(\frac{1}{c^{2} \xi^{\prime}(1)}-1\right)}\right)\left(\frac{1}{c\left(\frac{1}{c^{2} \xi^{\prime}(1)}-1\right)} \log \left(\frac{1}{c^{2} \xi^{\prime}(1)}\right)-c \xi^{\prime}(1)\right) \\
& =\left(\frac{c^{2} \xi^{\prime}(1)}{1-c^{2} \xi^{\prime}(1)}\right) \xi^{\prime}(1)\left[\left(\frac{1}{1-c^{2} \xi^{\prime}(1)}\right) \log \left(\frac{1}{c^{2} \xi^{\prime}(1)}\right)-1\right] .
\end{aligned}
$$

The next result is regarding a sufficient condition for optimality and will be used in the subsequent.

Lemma 5.3. Assume that $m, c>0$ solve (5.1.1). Let $\phi=m(1-t)+c$ and let $\eta$ be its formal conjugate. If $\eta \geq \xi$ on $[0,1]$, then $\phi$ and $\eta$ are optimal for $P$ and $D$.

Proof. Since $\eta$ satisfies (1.3.4), we have that $\eta$ is continuous, convex, and satisfies the boundary conditions in $\mathcal{K}_{\xi}$. We also have that $\phi^{2} \eta^{\prime \prime}=1$. By Fact 5.1 and since $\phi^{\prime \prime}=0$, we have that $\int \xi^{\prime \prime} \phi=\int \eta^{\prime \prime} \phi$. Thus, the result follows by Lemma 3.3 .

\subsubsection{Proof of Corollary 1.23.}

Proof. Assume that the optimal $\phi$ is in $R S B_{1}$, and write $\phi(t)=m_{*}(1-t)+c_{*}$. Let $\eta \in \mathcal{K}_{\xi}$ be the corresponding optimizer. By Theorem 1.10, Theorem 1.11, and 6.10, $\eta$ is the formal conjugate to $\phi$ given by (1.3.4). Since $\phi^{\prime}(0) \neq 0$, the natural boundary conditions from Theorem 1.11(d) imply that $\eta(0)=\xi(0)$ and $\eta^{\prime}(1)=\xi^{\prime}(1)$. Now claim (1) follows from Fact 5.1. Claims (2) and (3) follow from Theorem $1.11(c)$ after observing that 0 and 1 are contact points.

The following lemma justifies the appearance of the "pure-like or critical" condition appearing in the statement of Corollary 1.23 .

Lemma 5.4. Assume that $\xi \neq \xi_{S K}$. Let $m, c>0$ solve (5.1.1) and let $\eta$ be the formal conjugate to $\phi(t)=m(1-t)+c$. Then $\eta^{\prime \prime}(1) \geq \xi^{\prime \prime}(1)$ if and only if $\xi$ is pure-like or critical.

Proof. First we note that since $\xi \neq \xi_{S K}, \xi^{\prime \prime}(1)>\xi^{\prime}(1)$. Now by 1.3 .4$), \eta(t)=\xi(1)-R(t)$ so that

$$
\eta^{\prime \prime}(t)=-R^{\prime \prime}(t)=\frac{1}{(c+m(1-t))^{2}} .
$$

Thus, $\eta^{\prime \prime}(1) \geq \xi^{\prime \prime}(1)$ if and only if $c^{2} \leq \frac{1}{\xi^{\prime \prime}(1)}$. Let $y=c^{2} \xi^{\prime}(1)$, then by the master equation(5.1.2),

$$
\xi(1)=\frac{y}{1-y} \xi^{\prime}(1)\left(\frac{1}{1-y} \log \left(\frac{1}{y}\right)-1\right) .
$$

Since the function

$$
f(s)=\frac{s}{1-s} \xi^{\prime}(1)\left(\frac{1}{1-s} \log \left(\frac{1}{s}\right)-1\right)
$$

is increasing on $[0,1), c^{2} \leq \frac{1}{\xi^{\prime \prime}(1)}$ if and only if $f(y) \leq f\left(\frac{\xi^{\prime}(1)}{\xi^{\prime \prime}(1)}\right)$. Rewriting the last condition gives

$$
\begin{aligned}
\xi(1) & \leq \frac{\frac{\xi^{\prime}(1)}{\xi^{\prime \prime}(1)}}{1-\frac{\xi^{\prime}(1)}{\xi^{\prime \prime}(1)}} \xi^{\prime}(1)\left(\frac{1}{1-\frac{\xi^{\prime}(1)}{\xi^{\prime \prime}(1)}} \log \left(\frac{1}{\frac{\xi^{\prime}(1)}{\xi^{\prime \prime}(1)}}\right)-1\right) \\
& =\frac{\xi^{\prime}(1)^{2} \xi^{\prime \prime}(1)}{\left(\xi^{\prime \prime}(1)-\xi^{\prime}(1)\right)^{2}}\left[\log \left(\frac{\xi^{\prime \prime}}{\xi^{\prime}}\right)-\frac{\xi^{\prime \prime}-\xi^{\prime}}{\xi^{\prime \prime}}\right](1) .
\end{aligned}
$$


Since $\xi^{\prime \prime}(1)-\xi^{\prime}(1) \neq 0$, this is equivalent to

$$
\begin{aligned}
0 & \leq\left[\log \left(\frac{\xi^{\prime \prime}}{\xi^{\prime}}\right)-\frac{\xi^{\prime \prime}-\xi^{\prime}}{\xi^{\prime \prime}}-\xi \frac{\left(\xi^{\prime \prime}-\xi^{\prime}\right)^{2}}{\xi^{\prime 2} \xi^{\prime \prime}}\right](1) \\
& =\left[\log \left(\frac{\xi^{\prime \prime}}{\xi^{\prime}}\right)-\frac{\left(\xi^{\prime \prime}-\xi^{\prime}\right)\left(\xi\left(\xi^{\prime \prime}-\xi^{\prime}\right)+\xi^{\prime 2}\right)}{\xi^{\prime 2} \xi^{\prime \prime}}\right](1)=A B A\left(\nu^{\prime}, \nu^{\prime \prime}\right),
\end{aligned}
$$

where the last equality comes from the assumption that $\xi(1)=1$.

5.2. 1 RSB in $\mathbf{2}+\mathbf{p}$ models. In this subsection we will prove Theorem 1.25. We begin with the following observations. If we let $y=\frac{1}{c^{2} \xi^{\prime}(1)}$, we see that the master equation $(5.1 .2)$ is equivalent to

$$
\frac{\xi(1)}{\xi^{\prime}(1)}=\frac{1}{y-1}\left(\frac{y}{y-1} \log y-1\right)
$$

We have that $0<\frac{\xi(1)}{\xi^{\prime}(1)} \leq \frac{1}{2}$ with equality if and only if $\xi=\xi_{S K}$. Observe the following fact which follows by a standard calculus exercise.

Fact 5.5. There exists a unique solution $y_{\xi} \in[1, \infty)$ to the equation (5.2.1). The solution satisfies $y_{\xi}=1$ if and only if $\xi=\xi_{S K}$. Finally, if $y_{\xi}<1$, then (5.1.1) and (5.1.2) have unique positive solutions.

Proof. Consider $a:(1, \infty) \rightarrow \mathbb{R}$

$$
a(y)=\frac{1}{y-1}\left(\frac{y}{y-1} \log y-1\right)
$$

It is then a calculus exercise to show that

- $a$ is continuous and strictly decreasing on $(1, \infty)$

- $a(1+)=\frac{1}{2}$ and $a(+\infty)=0$

Therefore, $a$ is invertible, $a^{-1}:(0,1) \rightarrow \mathbb{R}_{+}$, and $a^{-1}$ is continuous. This proves the first two claims. For the third claim, note $y_{\xi}=1$ if and only if $\frac{\xi(1)}{\xi^{\prime}(1)}=\frac{1}{2}$ which it true if and only if $\xi=\xi_{S K}$.

With this in hand, we observe that one can rewrite the non-negative replicon eigenvalue and pure-like or critical conditions as upper and lower bounds on $y_{\xi}$ respectively. More precisely,

Lemma 5.6. Let $\xi \neq \xi_{S K}$. Then $\xi$ satisfies the "non-negative replicon eigenvalue" and "pure-like or critical" conditions if and only if

$$
\frac{\xi^{\prime \prime}(1)}{\xi^{\prime}(1)} \leq y_{\xi} \leq \frac{\xi^{\prime}(1)}{\xi^{\prime \prime}(0)}
$$

Proof. Let $m, c>0$ solve (5.1.1) and let $\eta$ be the formal conjugate to $\phi=m(1-t)+c$. Then

$$
\eta^{\prime \prime}(t)=\frac{1}{(m(1-t)+c)^{2}} .
$$

By definition, the "non-negative replicon eigenvalue" condition is that $\frac{1}{(m+c)^{2}} \geq \xi^{\prime \prime}(0)$. By (5.1.1) this is equivalent to $c^{2}\left(\xi^{\prime}(1)\right)^{2} \geq \xi^{\prime \prime}(0)$. Similarly, by Lemma 5.4 we have that the "pure-like or critical" condition is equivalent to $\frac{1}{c^{2}} \geq \xi^{\prime \prime}(1)$. Now by Lemma 5.2 and Fact $5.5, y_{\xi}=\frac{1}{c^{2} \xi^{\prime}(1)}$. The result immediately follows. 
For the remainder of this section we will be specifically addressing models of the form $\xi_{\mu}(t)=$ $\mu t^{2}+(1-\mu) t^{p}$ where $p \geq 3$ is fixed and $0 \leq \mu<1$. Then we refer to the point $y_{\xi}$ as $y_{\mu}$.

Define

$$
h_{\mu}=\frac{1}{\phi_{\mu}^{2}}-\xi_{\mu}^{\prime \prime}
$$

where $\phi_{\mu}(t)=c_{\mu}+m_{\mu}(1-t)$, and where $c, m$ are determined from $\mu$ by solving (5.1.1). Observe the following lemma.

Lemma 5.7. (root bound) Let $\mu \in[0,1]$ be such that $y_{\mu}$ satisfies 5.2.2). Then the equation $h_{\mu}(t)=0$ has at most two solutions in $(0,1)$.

Proof. We suppress the subscript $\mu$ for readability when convenient. Let $Z=\phi^{2} h=1-\phi^{2} \xi^{\prime \prime}$ and note that the roots of $Z$ and $h$ are the same. Observe that

$$
\phi^{2}(t)=(c+m(1-t))^{2}=c^{2}\left(1+\left(\frac{1}{c^{2} \xi^{\prime}(1)}-1\right)(1-t)\right)^{2}=C(\xi, y)\left(\frac{y}{y-1}-t\right)^{2}
$$

where $C(\xi, y)=\frac{(y-1)^{2}}{\xi^{\prime}(1) y}$. In particular, $Z_{\xi}(t)=1-C\left(\xi, y_{\xi}\right)\left(\frac{y_{\xi}}{y_{\xi}-1}-t\right)^{2} \xi^{\prime \prime}(t)$. Specializing to the case of $2+p$, with $\xi=\xi_{\mu}$, we have

$$
\begin{aligned}
Z_{\mu}(t) & =1-C\left(\xi_{\mu}, y_{\mu}\right)\left(\frac{y_{\mu}}{y_{\mu}-1}-t\right)^{2}\left(2 \mu+p(p-1)(1-\mu) t^{p-2}\right) \\
C\left(\xi_{\mu}, y\right) & =\frac{1}{2 \mu+p(1-\mu)} \frac{(y-1)^{2}}{y} .
\end{aligned}
$$

Now the plan is to expand $Z$ and apply Descartes' rules of signs. We begin by observing that by the non-negative replicon eigenvalue condition (i.e., $h(0) \geq 0$ ), we have that $Z(0) \geq 0$, and similarly by the pure-like or critical condition (i.e., that $h(1) \geq 0$ ), we have $Z(1) \geq 0$. Let $x=\frac{y}{y-1}$, which is positive since $y>1$, and expand $Z$ to find that

$$
\begin{aligned}
Z & =1-C \xi^{\prime \prime}(t)(x-t)^{2}=1-C \xi^{\prime \prime}(t)\left(x^{2}-2 x t+t^{2}\right) \\
& =Z(0)+C 2 \mu 2 x t-C 2 \mu t^{2}-C p(p-1)(1-\mu) t^{p-2} x^{2}+C p(p-1)(1-\mu) t^{p-1} 2 x-C p(p-1)(1-\mu) t^{p} .
\end{aligned}
$$

Since $Z(0) \geq 0$ we count at most three sign changes (checking the cases $p=3$ and $p=4$ separately), so that by Descartes' rule of signs there are at most three positive real roots. Since $Z(+\infty)=-\infty$ and $Z(1) \geq 0$, one of those roots must be in $[1, \infty)$. This proves the claim.

Now we have a lemma regarding the sign of $Z$ near 0 .

Lemma 5.8. Let $y_{\mu}$ satisfy 5.2 .2 . Then there is a $\delta>0$ such that $Z>0$ on $(0, \delta)$.

Proof. By (5.2.2), we see that $Z(0), Z(1) \geq 0$. Now for the first claim: If $Z(0)>0$ then the result follows by continuity. If $Z(0)=0$, then since $C, x \neq 0$, and since $Z(0)=0$ then you must have that $\mu \neq 0$. Then $Z(t)=4 C x \mu t+o(t)$ for $t \rightarrow 0$, so the result follows.

5.2.1. Proof of Theorem 1.25. Before we begin the proof, recall that we are considering models of the form $\xi_{\mu}(t)=\mu t^{2}+(1-\mu) t^{p}$ where $p \geq 3$ is fixed and $0 \leq \mu<1$. Recall also that we refer to $y_{\xi}$ as defined in Fact 5.5 as $y_{\mu}$. We now begin the proof.

Proof. The "only if" part of the theorem is implied by Corollary 1.23 . Now we show the "if" part.

To begin, let $m, c>0$ solve (5.1.1) and let $\eta$ be the formal conjugate to $\phi(t)=m(1-t)+c$. Assume that the "non-negative replicon eigenvalue" and the "pure-like or critical" conditions hold, i.e., assume that $\eta^{\prime \prime}(0) \geq \xi^{\prime \prime}(0)$ and that $\eta^{\prime \prime}(1) \geq \xi^{\prime \prime}(1)$. (See Lemma 5.4.) Our goal is to show that $\phi$ and $\eta$ are optimal. Recall that by Lemma 5.3, this will hold provided that the obstacle 
condition, $\eta \geq \xi$ for all $t \in[0,1]$, holds. We will prove this by studying the difference of the second derivatives, $h_{\mu}$ from $(5.2 .3)$.

We begin by observing that $h_{\mu}$ undergoes at most two sign changes in $(0,1)$. To see this, observe that by Lemma 5.6. $y_{\mu}$ satisfies (5.2.2) . The observation then follows by Lemma 5.7. Furthermore, by Lemma 5.8 we know that $h_{\mu}$ is positive on a neighborhood of 0 . The obstacle condition will now follow by a case analysis.

In the first case, $h_{\mu}$ does not change sign. Then, $h_{\mu} \geq 0$ on $(0,1)$ and hence $\eta-\xi$ is convex there. Since $\eta(0)=\xi(0)$ and $\eta^{\prime}(0)=\xi^{\prime}(0)$, it follows that $\eta \geq \xi$ on $[0,1]$.

In the second case, $h_{\mu}$ undergoes one sign change. Then, there exists $\delta \in(0,1)$ such that $h_{\mu}>0$ on $(0, \delta)$ and $h_{\mu} \leq 0$ on $[\delta, 1)$. Thus $\eta-\xi$ is convex on $(0, \delta)$ and by the same argument as above we conclude that $\eta \geq \xi$ on $[0, \delta]$. Continuing, we have that $\eta(\delta) \geq \xi(\delta)$ and $\eta(1) \geq \xi(1)$, and also that $\eta-\xi$ is concave on $[\delta, 1)$. It immediately follows that $\eta \geq \xi$ on $[\delta, 1]$ as required.

In the final case, $h$ undergoes two sign changes. Then, there exist $\delta_{1}, \delta_{2} \in(0,1)$ with $\delta_{1}<\delta_{2}$, and such that $h_{\mu}>0$ on $\left(0, \delta_{1}\right) \cup\left(\delta_{2}, 1\right)$ and $h \leq 0$ on $\left[\delta_{1}, \delta_{2}\right]$. Using the boundary data at 0 and 1 and that $\eta-\xi$ is convex on $\left(0, \delta_{1}\right) \cup\left(\delta_{2}, 1\right)$, we conclude that $\eta \geq \xi$ on $\left[0, \delta_{1}\right] \cup\left[\delta_{2}, 1\right]$. Continuing, we have that $\eta\left(\delta_{i}\right) \geq \xi\left(\delta_{i}\right)$ for $i=1,2$ and that $\eta-\xi$ is concave on $\left[\delta_{1}, \delta_{2}\right]$. It follows that $\eta \geq \xi$ on $\left[\delta_{1}, \delta_{2}\right]$ as required.

\section{Appendix}

In this appendix, we collect some basic results that are used throughout the paper. In Section 6.1. we explain the relation between the Crisanti-Sommers functional as defined in this paper and those occurring previously in the literature. In Section 6.2, we present basic properties of the sets $\mathcal{A}, \mathcal{C}$, and $\mathcal{K}_{h, \xi}$. In Section 6.3, we present basic properties of $S$ and D. In Section 6.4, we prove basic results regarding sequential $\Gamma$-convergence.

6.1. Reformulation of the Crisanti-Sommers functional . The Crisanti-Sommers variational problem is usually posed in a different form. Let $\mu \in \operatorname{Pr}[0,1]$, and define $q_{*}=\sup \operatorname{supp} \mu$. The spherical Crisanti-Sommers functional is sometimes defined as

$$
\tilde{\mathcal{P}}(\mu ; \beta, h, \xi)=\left\{\begin{array}{ll}
\frac{1}{2}\left(\int_{0}^{1} \beta^{2} \xi^{\prime}(s) \mu[0, s] d s+h^{2} \hat{\mu}(0)+\int_{0}^{q_{*}} \frac{d s}{\hat{\mu}(s)}+\log \left(1-q_{*}\right)\right) & q_{*}<1 \\
+\infty & q_{*}=1
\end{array} .\right.
$$

This functional is not lower semi-continuous in the weak-* topology. In the original work of CrisantiSommers, the functional is only defined for $q_{*}<1$. That the minimization problem is unchanged by replacing the functional above with $\mathcal{P}$ from $\sqrt{1.0 .1}$ can be seen by the following lemma. On the other hand, the functional $\mathcal{P}$ is lower semi-continuous. As the original functional was not defined for $q_{*}=1$, we call this functional the Crisanti-Sommers functional without ambiguity.

Lemma 6.1. For $q_{*}<1$, we have that

$$
\tilde{\mathcal{P}}_{\beta, h, \xi}(\mu)=\mathcal{P}_{\beta, h, \xi}(\mu) .
$$

Proof. To see this, observe that

$$
\int_{0}^{q_{*}} \frac{d s}{\int_{s}^{1} \mu[0, t] d t}+\log \left(1-q_{*}\right)=\int_{0}^{q} \frac{d s}{\int_{s}^{1} \mu[0, t] d t}+\log (1-q)=\int_{0}^{q} \frac{d s}{\int_{s}^{1} \mu[0, t] d t}-\frac{1}{1-s} d s
$$

for all $q \in\left[q_{*}, 1\right)$, so that

$$
\int_{0}^{q_{*}} \frac{d s}{\int_{s}^{1} \mu[0, t] d t}+\log \left(1-q_{*}\right)=\int_{0}^{1}\left(\frac{1}{\hat{\mu}(s)}-\frac{1}{1-s}\right) d s .
$$


(This last integral is well-defined as the integrand is non-negative.) Now if we recall that $\xi^{\prime}(0)=0$ and use the fundamental theorem and Fubini, we see that

$$
\int_{0}^{1} \xi^{\prime}(s) \mu[0, s] d s=\int_{0 \leq t \leq s \leq 1} \xi^{\prime \prime}(t) \mu[0, s] d s=\int_{0}^{1} \xi^{\prime \prime}(t) \int_{t}^{1} \mu[0, s] d s .
$$

Grouping then gives the result.

Corollary 6.2. $\mathcal{P}$ is lower semi-continuous and $\tilde{\mathcal{P}}$ is not lower semi-continuous in the weak-* topology.

Proof. That $\mathcal{P}$ is lower semi-continuous is an application of Fatou's lemma. To see that $\tilde{\mathcal{P}}$ is not lower semi-continuous, take

$$
\mu(d t)=\frac{1}{2 \sqrt{1-t}} d t
$$

and let $\mu_{n}=\frac{1}{2 \sqrt{1-t}} \mathbb{1}_{t \leq 1-\frac{1}{n}} d t+\left(\int_{1-\frac{1}{n}}^{1} \frac{1}{2 \sqrt{1-t}} d t\right) \delta_{1-\frac{1}{n}}$. Observe that $\mu_{n} \rightarrow \mu$ weakly. Furthermore, $\mu_{n}[0, t] \downarrow \mu[0, t]$ so that by the monotone convergence theorem, $\mathcal{P}\left(\mu_{n}\right) \rightarrow \mathcal{P}(\mu)$. Thus by Lemma 6.1. $\tilde{\mathcal{P}}\left(\mu_{n}\right) \rightarrow \mathcal{P}(\mu)$. Now we claim that $\mathcal{P}(\mu)<\infty$. Indeed, $\hat{\mu}(s)=\sqrt{1-s}$ so that

$$
0 \leq \frac{1}{\hat{\mu}(s)}-\frac{1}{1-s} \leq \frac{1}{\sqrt{1-s}} \in L^{1} .
$$

Thus $\liminf \tilde{\mathcal{P}}\left(\mu_{n}\right)<\infty$ while $\tilde{\mathcal{P}}(\mu)=\infty$.

6.2. On the sets $\mathcal{A}, \mathcal{C}$, and $\mathcal{K}_{h, \xi}$. In this section, we state some important and basic properties of the sets $\mathcal{A}, \mathcal{C}$, and $\mathcal{K}_{h, \xi}$.

6.2.1. On the set $\mathcal{A}$.

Lemma 6.3. Let $f \in L^{1}([0,1])$ be non-negative and positive on a set of positive Lebesgue measure. Then for every $C>0$, the set

$$
\left\{\nu \in \mathcal{A}: \int f(s) \nu[s, 1] d s \leq C\right\}
$$

is weak-* sequentially compact. In particular, on this set we have that

$$
\nu([0,1]) \leq \frac{C}{\int_{0}^{1} \int_{0}^{t} f(s) d s d t} .
$$

Proof. Write $d \nu=m(t) d t+c \delta_{1}$, then by Fubini we have that

$$
\int_{0}^{1} f(s) \nu[s, 1] d s=\int_{0 \leq s \leq t \leq 1} f(s) d \nu(t) d s=\int_{0}^{1} \int_{0}^{t} f(s) d s d \nu(t)=\int_{0}^{1} F(t) m(t) d t+c F(1)
$$

where $F(t)=\int_{0}^{t} f(s) d s$. By the Harris-FKG inequality,

$$
\int_{0}^{1} F(t) m(t) d t \geq \int_{0}^{1} F(t) d t \int_{0}^{1} m(t) d t
$$

so that

$$
\int_{0}^{1} f(s) \nu[s, 1] d s \geq \int_{0}^{1} F(t) d t \int_{0}^{1} m(t) d t+c F(1) \geq \int_{0}^{1} F(t) d t \cdot \nu([0,1]) .
$$

The sequential compactness result now follows from the mass bound above and the fact that $\mathcal{A}$ is weak-* sequentially closed in $\mathscr{M}$.

Lemma 6.4. The sets $\mathcal{C}$ and $\mathcal{A}$ are in bijective correspondence. This correspondence is given by $\phi(t)=\nu[t, 1]$. 
Proof. Define the map $\Psi: \phi(t) \mapsto-\phi^{\prime}(s) d s+\phi(1) \delta_{1}$. Since $\phi \in \mathcal{C}$ we know that $\phi^{\prime} \in L^{1}$ and has a cadlag, monotone decreasing version. Furthermore, $-\phi^{\prime} \geq 0$. Thus $\Psi: \mathcal{C} \rightarrow \mathcal{A}$. Then given $\phi \in \mathcal{C}$ we have that

$$
\phi(t)=\phi(1)-\int_{t}^{1} \phi^{\prime}(t) d t=\int_{t}^{1} d \nu
$$

where $\nu=\phi^{\prime}(s) d s+\phi(1) \delta_{1}$, so that $\Psi$ is injective. Now let $\nu \in \mathcal{A}$ and let $\phi(t)=\int_{t}^{1} d \nu$. By definition of $\nu, \phi \in C([0,1])$ and is non-increasing. Since $m(t)=\phi^{\prime}(t)$ is cadlag and monotone, $\phi$ is convex. Thus $\phi \in \mathcal{C}$ and $\Psi(\phi)=\nu$ so that the map $\Psi$ is surjective.

6.2.2. On the set $\mathcal{C}$.

Lemma 6.5. For every $C>0$, the set $E=\mathcal{C} \cap\left\{\phi:\|\phi\|_{\infty} \leq C\right\}$ is norm compact.

Proof. This is an application of Helly's selection theorem, Dini's theorem on the pointwise limit of continuous, monotone functions, and the fact that if $\phi \in \mathcal{C}$ then $\left\|\phi^{\prime}\right\|_{L^{1}} \leq\|\phi\|_{L^{\infty}}$.

Lemma 6.6. Let $f \in L^{1}([0,1])$ be non-negative and positive on a set of positive Lebesgue measure. Then for every $C>0$ the set

$$
S=\left\{\phi \in \mathcal{C}: \int f \phi \leq C\right\}
$$

is norm compact.

Proof. Observe first that it is closed. Now we claim that $S$ admits a uniform $L^{\infty}$ bound. Then the result will follow from Lemma 6.5.

To see the uniform $L^{\infty}$ bound, note that it is enough to bound $\phi(0)$ by monotonicity properties of $\mathcal{C}$. Now by concavity of $\phi$ and non-negativity of $f$, we have that

$$
\int f \phi \geq \int f(t)((1-t) \phi(0)+t \phi(1)) d t \geq \phi(0) \int f(t)(1-t) d t+\phi(1) \int f(t) t d t .
$$

Hence,

$$
\|\phi\|_{L^{\infty}}=\phi(0) \leq \frac{\int f \phi}{\int f(t)(1-t) d t}
$$

Given $\phi \in \mathcal{C}$, it is immediate that $\phi \in W^{1,1} \subset B V$. However, it may not be true that $\phi^{\prime} \in B V$. Thus, we must be careful with how we define the meaning of $\phi^{\prime}$ at the boundary points 0,1 . We call

$$
\begin{aligned}
& \phi^{\prime}(1)=\lim _{t \rightarrow 1^{-}} \frac{\phi(1)-\phi(t)}{1-t} \\
& \phi^{\prime}(0)=\lim _{t \rightarrow 0^{+}} \frac{\phi(t)-\phi(0)}{t} .
\end{aligned}
$$

Since $\phi$ is concave, both limits exist, though they may be infinite a priori. However, it is apparent from the definition of $\mathcal{C}$ that $\phi^{\prime}(0) \in \mathbb{R}$ and $\phi^{\prime}(1) \in \mathbb{R} \cup\{-\infty\}$.

On the other hand, as a result of Theorem 6.12, we can identify $\phi^{\prime \prime}=-\mu$ as distributions, where $\mu$ is a non-negative Radon measure on $(0,1)$. The next lemma relates the mass of this measure to the boundary values of $\phi^{\prime}$.

Lemma 6.7. Let $\phi \in \mathcal{C}$ and let $\phi^{\prime \prime}=-\mu$ as elements of $\mathcal{D}^{\prime}$, where $\mu$ is a non-negative Radon measure on $(0,1)$. Then,

$$
\mu((0,1))=\phi^{\prime}(0)-\phi^{\prime}(1) .
$$

Hence, $\phi \in \mathcal{C} \cap\left\{\phi^{\prime} \in B V\right\}$ if and only if $\phi^{\prime}(1)>-\infty$. 
We note that if $\phi \in \mathcal{C} \cap\left\{\phi^{\prime} \in B V\right\}$, then $\phi^{\prime}$ has well-defined trace at 0,1 , and $\left(\operatorname{Tr} \phi^{\prime}\right)(t)=\phi^{\prime}(t)$ for $t=0,1$.

Finally, we have the following useful approximation result.

Lemma 6.8. If $\phi \in \mathcal{C}$ and $\phi^{\prime}(1)=-\infty$, then there is a sequence $\left\{\phi_{n}\right\} \subset \mathcal{C}$ with $\phi_{n} \rightarrow \phi$ and $-\infty<\phi_{n}^{\prime}(1) \leq-n$.

6.2.3. On the set $\mathcal{K}_{h, \xi}$. Since $\eta \in \mathcal{K}_{h, \xi}$ is convex, we can define $\eta^{\prime}$ at the boundary points 0,1 by

$$
\begin{aligned}
& \eta^{\prime}(1)=\lim _{t \rightarrow 1^{-}} \frac{\eta(1)-\eta(t)}{1-t} \\
& \eta^{\prime}(0)=\lim _{t \rightarrow 0^{+}} \frac{\eta(t)-\eta(0)}{t} .
\end{aligned}
$$

It also follows from convexity that $\eta^{\prime \prime}=\mu$ as elements of $\mathcal{D}^{\prime}$, where $\mu$ is a non-negative Radon measure on $(0,1)$ (see Theorem 6.12).

The next lemma shows that in fact $\eta^{\prime} \in B V$. Hence, $\eta^{\prime}$ has well-defined trace at 0,1 and $\left(\operatorname{Tr} \eta^{\prime}\right)(t)=\eta^{\prime}(t)$ for $t=0,1$.

Lemma 6.9. Let $\eta \in \mathcal{K}_{h, \xi}$ and let $\eta^{\prime \prime}=\mu$ as elements of $\mathcal{D}^{\prime}$. Then $\eta^{\prime}(1) \leq \xi^{\prime}(1)$ and $\eta^{\prime}(0) \geq \xi^{\prime}(0)$. Hence, $\eta^{\prime} \in B V$ and

$$
\mu((0,1))=\eta^{\prime}(1)-\eta^{\prime}(0) \leq \xi^{\prime}(1)-\xi^{\prime}(0) .
$$

The next result describes a useful construction.

Lemma 6.10. Let $\sigma$ be a finite Radon measure on $(0,1)$ and let $a, b \in \mathbb{R}$. Then the boundary value problem

$$
\left\{\begin{array}{l}
\eta^{\prime \prime}=\sigma \\
\eta^{\prime}(0)=a \\
\eta(1)=b
\end{array}\right.
$$

has a unique solution in the class $\eta \in C([0,1]) \cap\left\{\eta^{\prime} \in B V\right\}$.

6.2.4. An integration by parts lemma.

Lemma 6.11. (Integration by parts) Suppose that $\eta, \phi \in C([0,1]) \cap\left\{\phi^{\prime} \in B V\right\}$. Then

$$
\left(\eta^{\prime \prime}, \phi\right)=\left.\eta^{\prime} \phi\right|_{0} ^{1}-\left.\eta \phi^{\prime}\right|_{0} ^{1}+\left(\eta, \phi^{\prime \prime}\right) \text {. }
$$

Proof. Applying the integration by parts theorem from [14, Section 5.3] along with a straightforward approximation argument, we conclude that

$$
\begin{aligned}
& \int_{(0,1)} \phi d \eta^{\prime \prime}=-\int_{(0,1)} \eta^{\prime} \phi^{\prime} d x+\left.\phi \eta^{\prime}\right|_{0} ^{1} \\
& \int_{(0,1)} \eta d \phi^{\prime \prime}=-\int_{(0,1)} \eta^{\prime} \phi^{\prime} d x+\left.\phi^{\prime} \eta\right|_{0} ^{1} .
\end{aligned}
$$

Subtracting these gives the desired result.

6.2.5. Alexandrov's theorem. The following is a modification of a theorem of Alexandrov.

Theorem 6.12. [14 (Alexandrov) Let $f:(0,1) \rightarrow \mathbb{R}$ be convex, then $f^{\prime \prime}=\mu$ as elements of $\mathcal{D}^{\prime}$ where $\mu$ is a Radon measure on $(0,1)$. 


\subsection{Square Roots of Positive Measures.}

Lemma 6.13. We have the equality

$$
S(\mu)=\inf _{\phi \geq 0} \int \phi d \mu+\int \frac{1}{\phi} d x=\inf _{\substack{\phi \geq 0 \\ \phi \text { u.s.c. } \\ \phi \in L^{\infty}}} \int \phi d \mu+\int \frac{1}{\phi} d x .
$$

Proof. Begin by noting that

$$
\inf _{\phi \geq 0} \int \phi \mu_{a c} d x+\int \frac{1}{\phi} d x \geq 2 \int \sqrt{\mu_{a c}}
$$

by the arithmetic-geometric inequality so that

$$
S(\mu) \leq \inf _{\substack{\phi \geq 0 \\ \phi u . s . c . \\ \phi \in L^{\infty}}} \int \phi d \mu+\int \frac{1}{\phi} d x \leq \inf _{\substack{\phi \geq 0 \\ \phi \in C^{\infty}([0,1])}} \int \phi d \mu+\int \frac{1}{\phi} d x=: G(\mu)
$$

Write $\mu=f d x+d \nu$ with $\nu \perp \mathcal{L}$ where $\mathcal{L}$ denotes the Lebesgue measure. Let $f_{\epsilon}$ be a smooth, non-negative $L^{1}$ approximation to $f$. Find a Hahn decomposition $I=A \sqcup B$ such that $\nu(A)=0$, $\mathcal{L}(B)=0$. Given any $U \supset B$ open, $m, \lambda>0$, define

$$
\phi=\phi_{U, \epsilon, m, \lambda}= \begin{cases}\frac{1}{\sqrt{f_{\epsilon}}+m} & U^{c} \\ \lambda \wedge \frac{1}{\sqrt{f_{\epsilon}}+m} & U\end{cases}
$$

for $m>0$. Note that $\phi$ is upper semi-continuous, non-negative, and satisfies the bound

$$
\phi \leq \frac{1}{m} \wedge \frac{1}{\sqrt{f_{\epsilon}}+m} .
$$

So, we get that

We have that

$$
G(\mu) \leq \int \phi d \mu+\int \frac{1}{\phi} d x
$$

$\int \phi d \mu=\int \phi f d x+\int \phi d \nu=\int \phi\left(f-f_{\epsilon}\right) d x+\int \phi\left(f_{\epsilon} d x+d \nu\right) \leq \frac{1}{m}\left\|f-f_{\epsilon}\right\|_{L^{1}}+\int \phi\left(f_{\epsilon} d x+d \nu\right)$.

Also we have that

$$
\int \phi\left(f_{\epsilon} d x+d \nu\right)=\int \phi f_{\epsilon} d x+\int_{B} \phi d \nu \leq \int \sqrt{f_{\epsilon}} d x+\lambda \nu(U)
$$

and that

$$
\int \frac{1}{\phi} d x=\int_{I \backslash U}\left(\sqrt{f_{\epsilon}}+m\right) d x+\int_{U} \frac{1}{\lambda} \vee\left(\sqrt{f_{\epsilon}}+m\right) d x \leq \int \sqrt{f^{\epsilon}} d x+m+\frac{1}{\lambda} \mathcal{L}(U) .
$$

Adding up, we get that

$$
G(\mu) \leq 2 \int \sqrt{f_{\epsilon}} d x+\lambda \nu(U)+\frac{1}{\lambda} \mathcal{L}(U)+m+\frac{1}{m}\left\|f-f_{\epsilon}\right\|_{L^{1}}
$$

and optimizing in $m$ and $\lambda$ gives

$$
G(\mu) \leq 2 \int \sqrt{f_{\epsilon}} d x+2 \sqrt{\nu(U) \mathcal{L}(U)}+2\left\|f-f_{\epsilon}\right\|_{L^{1}}^{1 / 2} .
$$

Taking $U \downarrow B$ and then $f_{\epsilon} \rightarrow f$ in $L^{1}$ proves that $G(\mu) \leq 2 \int \sqrt{f} d x=2 \int \sqrt{\mu_{a c}}$.

Therefore, we have shown that $S \leq G \leq 2 \int \sqrt{\mu_{a c}} \leq S$ as desired.

Corollary 6.14. The functional $S: \mathscr{M}_{+} \rightarrow \mathbb{R}$ is upper semi-continuous in the weak-* and norm topologies on $\mathscr{M}_{+}$. 
Proof. We proved above that

$$
S(\mu)=\inf _{\substack{\phi \geq 0 \\ \phi u . s . c . \\ \phi \in L^{\infty}}} \int \phi d \mu+\int \frac{1}{\phi} d x .
$$

Note that for a fixed such $\phi$, the functional $\mu \rightarrow \int \phi d \mu$ is upper semi-continuous in the norm and weak-* topologies. Indeed, the assumptions on $\phi$ imply that

$$
\int \phi d \mu=\inf _{\substack{\psi \geq \phi \\ \psi \in C[0,1]}} \int \psi d \mu .
$$

Therefore it can be written as the point-wise infimum of continuous functionals, hence it is upper semi-continuous. As a result $S(\mu)$ is the point-wise infimum of upper semi-continuous functions so that it is upper semi-continuous.

Corollary 6.15. The functional $D: \mathcal{K}_{h, \xi} \rightarrow \mathbb{R}$ is upper semi-continuous in the norm topology.

6.4. Basic Theorems in $\Gamma$-convergence. In the following, let $X$ be a topological space and let $F_{\beta}, F: X \rightarrow[-\infty, \infty]$. Recall that the family of functions $F_{\beta}$ is said to be sequentially equi-coercive if for every $C>0$ there is a sequentially compact set $K \subset X$ such that for every $\beta$,

$$
\left\{F_{\beta} \leq C\right\} \subset K \text {. }
$$

Furthermore recall that a sequence of functions $G_{\beta} \rightarrow G$ continuously if for every $x_{\beta} \rightarrow x$, $G_{\beta}\left(x_{\beta}\right) \rightarrow G(x)$.

Theorem 6.16. (Fundamental Theorem of $\Gamma$-convergence) Suppose $F$ is not identically infinite. Suppose further that $F_{\beta} \stackrel{\Gamma}{\rightarrow} F, F_{\beta}$ are sequentially equi-coercive, $F$ has a unique minimizer, $x_{\beta}$ are minimizers of $F_{\beta}$, and $x$ is the unique minimizer of $F$. Then

$$
\lim _{\beta \rightarrow \infty} \min F_{\beta}=\min F
$$

and $x_{\beta} \rightarrow x$.

Proof. Without loss of generality, $F_{\beta}, F$ are finite. Fix a sequence $\beta_{n} \rightarrow \infty$. By the $\Gamma-\lim \sup$ inequality, we have that there is a sequence $y_{n} \rightarrow x$

$$
F(x) \geq \limsup F_{\beta_{n}}\left(y_{n}\right) \geq \limsup \inf _{z \in X} F_{\beta_{n}}(z) \geq \limsup F_{\beta_{n}}\left(x_{\beta_{n}}\right) .
$$

As a result, there is a $C$ such that $F_{\beta_{n}}\left(x_{\beta_{n}}\right) \leq C$. By sequential equi-coercivity, this implies that $\left\{x_{\beta_{n}}\right\} \subset K$ for some sequentially compact set. In particular, there is a further subsequence such that $x_{\beta_{n_{k}}} \rightarrow y$ for some $y \in X$. Then

$$
F(x) \geq \liminf F_{\beta_{n_{k}}}\left(x_{\beta_{n_{k}}}\right) \geq F(y)
$$

by the above inequalities and the $\Gamma-\lim$ inf inequality. Then since $x$ is the unique minimizer, $x=y$. Thus by the subsequence principle, $x_{\beta_{n}} \rightarrow x$.

The proof of the following is self-evident.

Theorem 6.17. (Stability under continuous perturbations) Suppose that $F_{\beta} \stackrel{\Gamma}{\rightarrow} F$ and that $G_{\beta} \rightarrow G$ continuously. Then $F_{\beta}+G_{\beta} \stackrel{\Gamma}{\rightarrow} F+G$. 


\section{REFERENCES}

[1] Antonio Auffinger and Gerard Ben Arous. Complexity of random smooth functions on the high-dimensional sphere. Ann. Probab., 41(6):4214-4247, 2013.

[2] Antonio Auffinger and Wei-Kuo Chen. On properties of parisi measures. Probability Theory and Related Fields, 161(3):817-850, 2014.

[3] Andrea Braides. $\Gamma$-convergence for beginners, volume 22 of Oxford Lecture Series in Mathematics and its Applications. Oxford University Press, Oxford, 2002.

[4] Haim Brézis, Lous Nirenberg, and Guido Stampacchia. A remark on Ky Fan's minimax principle. Boll. Un. Mat. Ital. (4), 6:293-300, 1972.

[5] Luis A. Caffarelli. The obstacle problem revisited. Journal of Fourier Analysis and Applications, 4(4):383-402, 1998.

[6] Luis A. Caffarelli and Avner Friedman. The obstacle problem for the biharmonic operator. Annali della Scuola Normale Superiore di Pisa - Classe di Scienze, 6(1):151-184, 1979.

[7] W.-K. Chen and A. Sen. Parisi formula, disorder chaos and fluctuation for the ground state energy in the spherical mixed p-spin models. ArXiv e-prints, December 2015.

[8] Wei-Kuo Chen. The Aizenman-Sims-Starr scheme and Parisi formula for mixed $p$-spin spherical models. Electron. J. Probab., 18:no. 94, 14, 2013.

[9] Giovanni Cimatti. The constrained elastic beam. Meccanica-J. Italian Assoc. Theoret. Appl. Mech., 8:119-124, 1973.

[10] Andrea Crisanti and Luca Leuzzi. Spherical $2+p$ spin-glass model: An exactly solvable model for glass to spin-glass transition. Phys. Rev. Lett., 93:217203, Nov 2004.

[11] Andrea Crisanti and Luca Leuzzi. Amorphous-amorphous transition and the two-step replica symmetry breaking phase. Phys. Rev. B, 76:184417, Nov 2007.

[12] Andrea Crisanti and Hans Jürgen Sommers. The spherical $p$-spin interaction spin glass model: the statics. Zeitschrift für Physik B Condensed Matter, 87(3):341-354, 1992.

[13] Gianni Dal Maso. An introduction to $\Gamma$-convergence. Progress in Nonlinear Differential Equations and their Applications, 8. Birkhäuser Boston, Inc., Boston, MA, 1993.

[14] Lawrence C. Evans and Ronald F. Gariepy. Measure theory and fine properties of functions. Studies in Advanced Mathematics. CRC Press, Boca Raton, FL, 1992.

[15] Aukosh Jagannath and Ian Tobasco. Some properties of the phase diagram for mixed p-spin glasses. Probability Theory and Related Fields, to appear.

[16] David Kinderlehrer and Guido Stampacchia. An introduction to variational inequalities and their applications, volume 31 of Classics in Applied Mathematics. Society for Industrial and Applied Mathematics (SIAM), Philadelphia, PA, 2000. Reprint of the 1980 original.

[17] Scott Kirkpatrick, C. D. Gelatt, and Mario P. Vecchi. Optimization by simulated annealing. Science, 220(4598):671-680, 1983.

[18] Vincent Krakoviack. Comment on "spherical $2+p$ spin-glass model: An analytically solvable model with a glass-to-glass transition". Phys. Rev. B, 76:136401, Oct 2007.

[19] Elliott H. Lieb and Michael Loss. Analysis, volume 14 of Graduate Studies in Mathematics. American Mathematical Society, Providence, RI, second edition, 2001.

[20] Olivier C. Martin, Rémi Monasson, and Riccardo Zecchina. Statistical mechanics methods and phase transitions in optimization problems. Theoretical Computer Science, 265(12):3 - 67, 2001. Phase Transitions in Combinatorial Problems.

[21] Marc Mézard, Giorgio Parisi, and Miguel Angel Virasoro. Spin glass theory and beyond, volume 9. World scientific Singapore, 1987.

[22] Louis Nirenberg. Topics in nonlinear functional analysis, volume 6 of Courant Lecture Notes in Mathematics. New York University, Courant Institute of Mathematical Sciences, New York; American Mathematical Society, Providence, RI, 2001. Chapter 6 by E. Zehnder, Notes by R. A. Artino, Revised reprint of the 1974 original.

[23] Dmitry Panchenko. The Sherrington-Kirkpatrick model. Springer, 2013.

[24] Sylvia Serfaty. Coulomb gases and Ginzburg-Landau vortices. Zurich Lectures in Advanced Mathematics. European Mathematical Society (EMS), Zürich, 2015.

[25] Eliran Subag. The complexity of spherical p-spin models - a second moment approach. ArXiv e-prints, April 2015.

[26] Michel Talagrand. Free energy of the spherical mean field model. Probab. Theory Related Fields, 134(3):339-382, 2006. 
(Aukosh Jagannath) Courant Institute of Mathematical Sciences, 251 Mercer St. NY, NY, USA, 10012 E-mail address: aukosh@cims.nyu.edu

(Ian Tobasco) Courant Institute of Mathematical Sciences, 251 Mercer St. NY, NY, USA, 10012

E-mail address: tobasco@cims.nyu.edu 